\title{
REVISITING AND EXTENDING INTERFACE PENALTIES FOR MULTI-DOMAIN SUMMATION-BY-PARTS OPERATORS
}

\author{
Mark H. Carpenter, * Jan Nordström ${ }^{\dagger}$ David Gottlieb ${ }^{\ddagger \S}$
}

\begin{abstract}
A general interface procedure is presented for multi-domain collocation methods satisfying the summationby-parts (SBP) spatial discretization convention. Unlike more traditional operators (e.g. FEM) applied to the advection-diffusion equation, the new procedure penalizes the solution and the first $p$ derivatives across the interface. The combined interior/interface operators are proven to be pointwise stable, and conservative, although accuracy deteriorates for $p \geq 2$. Penalties between two different sets of variables are compared (motivated by FEM primal and flux formulations), and are shown to be equivalent for certain choices of penalty parameters. Extensive validation studies are presented using two classes of high-order SBP operators: 1) central finite difference, and 2) Legendre spectral collocation.
\end{abstract}

summation-by-parts (SBP), high-order finite difference, finite element, DG, numerical stability, interface conditions, conservation

Applied and Numerical Mathematics

${ }^{*}$ Computational Aerosciences Branch, NASA Langley Research Center, Hampton, VA 23681-2199. Work performed in part, while in residence at TU Delft, Delft, The Netherlands. E-mail: mark.h.carpenter@nasa.gov.

$\dagger^{\dagger}$ Computational Physics Department, FOI SE-16490, Stockholm, Sweden; and the Department of Information Technology, Uppsala, SE75105, Uppsala, Sweden; The Department of Aeronautical and Vehicle Engineering, KTH - The Royal Institute of Technology, SE-10044, Stockholm, Sweden; E-mail: Jan.Nordstrom@foi.se.

${ }^{\ddagger}$ Formerly, Division of Applied Mathematics, Brown University, Providence, RI 02912.

$\S$ David Gottlieb passed away during the final revision of this paper. He left an indelible mark on our lives, and will be greatly missed. 


\section{Introduction}

High order finite difference methods (HOFDM) are ideally suited for simulations of complex physics requiring high fidelity solutions, and for a simple reason: efficiency. The extension of HOFDM to complex domains has proven difficult to accomplish, however. Multi-domain HOFDM are natural candidates for extension, but are fraught with the complexity of interface coupling procedures. Specifically, they must address the interface accuracy constraints, as well as contend with coupled stability conditions and interface conservation issues. Many ad hoc procedures have been developed to accommodate multi-domain methods, including conforming, nonconforming, and overset/overlapping procedures, (e.g. see Chesshire and Henshaw [10]). To enhance robustness, however, such techniques frequently require reduction in the order of accuracy at the interface and/or inclusion of specialized dissipation terms.

A simple solution to the multi-domain problem for HOFDM appears in the work of Carpenter, Nordström, Gottlieb[8] (henceforth referred to as CNG). Motivated by the recent popularity/success of Discontinuous Galerkin Finite Element methods (DGFEM), and the internal penalty approaches, CNG combine summation-by-parts (SBP) operators $[31,6,12,7,15,16,1,23,24,33,13]$ within each domain, with a penalty technique at the interface. The formulation requires only weak grid continuity at the interface $\left(C_{0}\right.$ : matching but not necessarily smooth), and is applicable to any operator that satisfies a SBP property. It maintains formal accuracy through the interface, is conservative and maintains a bounded semi-discrete energy estimate consistent with the underlying SBP operators. Similar developments within the nodal spectral element community are reported by Hesthaven et al. [17, 18, 19].

Although the CNG formulation is a powerful technique for coupling subdomains, it is not a general approach. Indeed, penalties could have been introduced in the original work using any smooth, collocated interface variable. A general methodology is advantageous because it allows greater flexibility in the construction of stable, accurate and conservative interface operators. (For example, one target application is the construction of block interface coupling operators needed for the newly developed energy stable WENO (ESWENO) schemes [35].) Thus, our objective is to derive general penalties between adjoining subdomains each discretized by a SBP operator. Herein, we present the framework for imposing penalties on the solution and first $p$ interface derivatives. The new penalties are constructed by first parameterizing all possible combinations of difference terms available at the interface. Energy methods are then used to constrain the parameters such that $L_{2}$ and pointwise stability, and conservation are achieved at the interface.

The newly derived penalties are formulated in two sets of variables, reminiscent of the primal and flux formulations commonly used in DGFEM for elliptic and parabolic equations. ${ }^{1}$ Both formulations are shown to be consistent, stable and conservative for the linear, constant coefficient, scalar advection diffusion equation. Furthermore, they are shown to be related; i.e. the flux formulation can be implemented using the primal variables if the interface penalties coefficients are allowed to vary with grid density. Numerous free parameters remain in both formulations even after stability and conservation constraints are imposed, and judicious choices for these parameters yield formulations analogous to popular schemes found in the FEM literature.

The construction of penalties from higher order derivatives is not without drawbacks. Indeed, it is shown that the formal accuracy suffers if terms involving second derivatives are included in the penalties. Nevertheless, these terms are retained because they allow the construction of richer penalties, and because empirical evidence suggests that the theoretical accuracy estimates may not always be sharp.

This paper is organized as follows. In section 2, the "general" approach for coupling adjoining SBP operators is presented, that includes penalties constructed from the solution and the first $p$ derivatives. Next, it is shown that the penalties can be formulated in two distinct sets of variables, although the resulting formulations are closely related. Section 3 presents a derivation of the theoretical accuracy of the new penalties, in the context of HOFDM. Section 4 identifies specific values for the penalty parameters that produce schemes analogous to several commonly used schemes. In section 5, numerical examples are presented for a variety of SBP operators. A discussion and conclusions are presented in sections 6 and 7 , respectively.

\footnotetext{
${ }^{1}$ The flux formulation is implemented on a transformed set of equations obtained by expressing the second-order elliptic (parabolic) scalar equation as a system of first-order equations. The resulting interface variables differ from those used in the conventional "primal" approach. See Arnold et al. [3] for a detailed description and comparison of the two approaches. Note that both weak-form FEM approaches differ from the strong-form collocation approach commonly used by HOFDM. See Carpenter et al. [9] for a comparison of weak- and strong-form approaches.
} 


\section{The semi-discrete problem}

\subsection{Definition of Stability}

Consider the continuous linear initial boundary value problem

$$
\begin{aligned}
w_{t} & =E w+F(x, t) & & , x \in \Omega & , t \geq 0, \\
w & =f(x) & & , x \in \Omega \quad & , t=0, \\
L_{C} w & =g(t) & & , x \in \Gamma & , t \geq 0,
\end{aligned}
$$

where $E$ is the differential operator and $L_{C}$ is the continuous boundary operator. The initial function $f$, the forcing function $F$, and the boundary term $g$ are the data for the problem.

Next, consider the semi-discrete counterpart of (1)

$$
\begin{array}{rlrlrl}
\left(w_{j}\right)_{t} & =\mathcal{E} w_{j}+F_{j}(t) & & , x_{j} \in \Omega & & , t \geq 0, \\
w_{j} & =f_{j} & & , x_{j} \in \Omega & , t=0, \\
L_{D} w_{j} & =g(t) & & , x_{j} \in \Gamma & , t \geq 0,
\end{array}
$$

where $\mathcal{E}$ is the difference operator approximating the differential operator $E, L_{D}$ is the discrete boundary operator, and $f_{j}, F_{j}$ and $g$ are the discrete initial function, forcing function, and boundary data, respectively.

Definition 1 The problem (2) is stable, for $F_{j}(t)=g=0$ and a sufficiently fine mesh, if the solution $w_{j}$ satisfies

$$
\frac{d}{d t}\|w\|_{\mathcal{P}}^{2} \leq 0
$$

where $\|\cdot\|_{\mathcal{P}}$ is a suitably defined discrete norm, such as a weighted $L_{2}$

$$
\|w\|_{\mathcal{P}}^{2}=w^{T} \mathcal{P} w
$$

Note that this restrictive definition of stability does not allow for temporal solution growth, as would be necessary to bound arbitrary source terms $F_{j}$ or boundary data $g(t)$. It is, however, well suited for establishing the stability of a numerical interface: our primary goal.

\subsection{Discrete SBP Operators}

Define the $\mathcal{P}$ weighted inner product $(v, w)_{\mathcal{P}}$ between two vectors, and let $U$ and $\mathcal{D} \mathbf{U}$ be the discrete approximations of the scalar quantities $u$ and $u_{x}$. The approximation $\mathcal{D} \mathbf{U}$ of the first derivative

$$
\mathcal{D} \mathbf{U}=\mathcal{P}^{-1} \mathcal{Q} \mathbf{U}, \quad \mathcal{P} \mathbf{u}_{\mathbf{x}}-\mathcal{Q} \mathbf{u}=\mathcal{P} T_{e 1}, \quad\left|T_{e 1}\right|=\mathcal{O}\left(\Delta x^{i}, \Delta x^{b}\right)
$$

satisfies the SBP rule

$$
\begin{gathered}
(\mathbf{U}, \mathcal{D} \mathbf{V})_{\mathcal{P}}=U_{n} V_{n}-U_{0} V_{0}-(\mathcal{D} \mathbf{U}, \mathbf{V})_{\mathcal{P}} \\
(\mathbf{U}, \mathbf{V})_{\mathcal{P}}=\mathbf{U}^{T} \mathcal{P} \mathbf{V}, \quad \mathcal{P}=\mathcal{P}^{T}, \quad \mathcal{Q}+\mathcal{Q}^{T}=\mathcal{B}, \quad \mathcal{B}=\operatorname{diag}[-1,0, . ., 0,1],
\end{gathered}
$$

$0<p_{\min } \Delta x\|\mathcal{I}\| \leq\|\mathcal{P}\| \leq p_{\max } \Delta x\|\mathcal{I}\| .{ }^{2}$ The matrix $\mathcal{P}$ is symmetric positive definite, the matrix $\mathcal{Q}$ is skew-symmetric with the exception of boundary points.

A second derivative operator can be obtained by applying any two first derivative operators, or by constructing the operator directly. Both techniques are suitable for spectral collocation. In HOFDM, using two consecutive nondissipative first derivatives, results in a second derivative operator that is unnecessarily wide and inaccurate and can

\footnotetext{
${ }^{2}$ Not to be confused with the vector norm, the nomenclature $\|\mathcal{P}\|$ here refers to a suitable matrix norm such as Frobenius.
} 
lead to odd-even mode decoupling. The operator, however, often leads to stability if varying coefficients are considered. Forming the HOFDM second derivative directly requires an operator with the following properties,

$$
\begin{gathered}
\mathcal{D}^{2} \mathbf{U}=\mathcal{P}^{-1} \mathcal{R}_{2} \mathbf{U}, \quad \mathcal{P} \mathbf{u}_{\mathbf{x x}}-\mathcal{R}_{2} \mathbf{u}=\mathcal{P} \mathbf{T}_{\mathbf{e} \mathbf{2}}, \quad \mathbf{T}_{\mathbf{e} \mathbf{2}}=\mathcal{O}\left(\Delta x^{i}, \Delta x^{b}\right), \\
\mathcal{R}_{2}=\left(-\mathcal{S}^{T} \mathcal{M}+\mathcal{B}\right) \mathcal{S},
\end{gathered}
$$

as suggested in reference [8] (see also [25]). The matrix $\mathcal{B}$ is given in (6), while the matrix $\mathcal{M}$ must be positive definite.

The matrix $\mathcal{S}$ is nearly diagonal with a discrete representation of the first derivative on the first and last rows,

$$
\{\mathcal{S} u\}_{0}=\{\mathcal{D} u\}_{0}=u_{x}\left(x_{0}, t\right)+T_{e 3}, \quad\{\mathcal{S} u\}_{n}=\{\mathcal{D} u\}_{n}=u_{x}\left(x_{n}, t\right)+T_{e 3},
$$

where $\left|T_{e 3}\right|=\mathcal{O}\left(\Delta x^{r}\right)$ and

$$
\mathcal{S}=\frac{1}{\Delta x}\left(\begin{array}{ccccccc}
s_{00} & s_{01} & s_{02} & s_{03} & \cdots & & \\
0 & 1 & 0 & & & & \\
& 0 & 1 & 0 & & & \\
& & \ddots & \ddots & \ddots & & \\
& & & 0 & 1 & 0 & \\
& & & & 0 & 1 & 0 \\
& & \cdots & s_{n n-3} & s_{n n-2} & s_{n n-1} & s_{n n}
\end{array}\right) \text {. }
$$

The second derivative defined in (7) and (8) satisfies a modified SBP rule. We have

$$
\left(\mathbf{U}, \mathcal{D}^{2} V\right)_{\mathcal{P}}=\mathbf{U}_{n}\{\mathcal{D} \mathbf{V}\}_{n}-\mathbf{U}_{0}\{\mathcal{D} \mathbf{V}\}_{0}-(\mathcal{S} \mathbf{U})^{T} M(\mathcal{S} \mathbf{V})
$$

The derivatives on the boundaries of $\mathcal{S}$ coincide with those found at the boundaries of the discrete operator $\mathcal{D}=\mathcal{P}^{-1} \mathcal{Q}$. [See equation (4)].

The notation $\left|T_{e 1}\right|,\left|T_{e 2}\right|=\mathcal{O}\left(\Delta x^{i}, \Delta x^{b}\right)$ and $\left|T_{e 3}\right|=\mathcal{O}\left(\Delta x^{r}\right)$ denotes that the approximation of the differential operator is accurate to order $i$ in the interior of the domain, to order $b$ at the boundary and that the approximation of the boundary conditions is accurate to order $r$. The relation between the different orders of accuracy, i.e., $i, b, r$ is discussed in section (4) below, where formal proofs of accuracy are given for HOFDM.

\section{3 "General" Primal-Form Interface Penalties}

Consider the constant coefficient, linear Burgers' equation

$$
\begin{array}{rlrlrl}
u_{t}+a u_{x} & =\epsilon u_{x x}+F(x, t) & & , t \geq 0 & ,-1 \leq x \leq 1, \\
u(x, 0) & =f(x) & & , t=0 \quad,-1 \leq x \leq 1, \\
\gamma u(-1, t)-\epsilon u_{x}(-1, t) & =g_{-1}(t) & = & L_{-1}(u) & , t \geq 0 & , x=-1, \\
\zeta u(+1, t)+\epsilon u_{x}(+1, t) & =g_{+1}(t) & = & L_{+1}(u) & , t \geq 0 & , x=+1, \\
0 \leq a+2 \zeta \quad & ; 0 \leq-a+2 \gamma . &
\end{array}
$$

with constants $a$ and $\epsilon(0<\epsilon)$. A detailed description of the wellposedness of this equation is omitted, but can be found in Carpenter et. al [9].

We are interested in the stability, conservation and accuracy of new interface treatments. For clarity of presentation (and without loss of generality), we shall neglect the source terms, and the treatment of the physical boundary conditions in the subsequent derivations. This means that we will consider stability of equations (9) and (10). Strong stability can easily be obtained by a proper treatment of the outer boundary conditions, but is not the focus of this paper. We are mainly interested in the interface treatment.

Assume that two adjoining SBP spatial operators support the first $p$ derivatives on their respective domains. A collocation approximation of the equation (9), motivated by the FEM proposed by Baumann and Oden [4], that includes penalties on the solution and the first $p$ derivatives is given by

$$
\begin{aligned}
\mathcal{P}_{l} \mathbf{U}_{t}+a \mathcal{P}_{l} \mathcal{D}_{l} \mathbf{U} & =\epsilon \mathcal{P}_{l} \mathcal{D}_{l} \mathcal{D}_{l} \mathbf{U}+\Sigma_{m=0}^{p} \Sigma_{k=0}^{p} l_{m k}\left[\left(\mathcal{D}_{l}^{k} U\right)_{i}-\left(\mathcal{D}_{r}^{k} V\right)_{i}\right]\left[\mathcal{D}_{l}^{m}\right]^{T} \mathbf{e}_{\mathbf{i}_{-}} \\
\mathbf{U}(x, 0) & =\mathbf{0} \\
\mathcal{P}_{r} \mathbf{V}_{t}+a \mathcal{P}_{r} \mathcal{D}_{r} \mathbf{V} & =\epsilon \mathcal{P}_{r} \mathcal{D}_{r} \mathcal{D}_{r} \mathbf{V}+\Sigma_{m=0}^{p} \Sigma_{k=0}^{p} r_{m k}\left[\left(\mathcal{D}_{r}^{k} U\right)_{i}-\left(\mathcal{D}_{r}^{k} V\right)_{i}\right]\left[\mathcal{D}_{r}^{m}\right]^{T} \mathbf{e}_{\mathbf{i}_{+}} \\
\mathbf{V}(x, 0) & =\mathbf{0}
\end{aligned}
$$




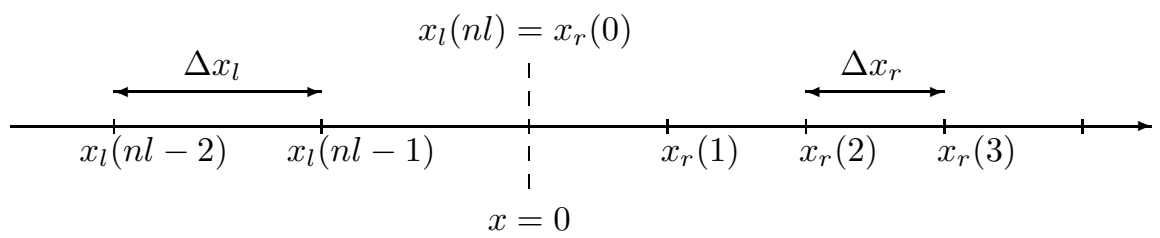

Figure 1: The mesh close to the interface at $x=0$.

The variables in the left (subscript $l)\left[x_{l}(0)=-1, x_{l}(n l)=0\right]$ and right (subscript $\left.r\right)\left[x_{r}(0)=0, x_{r}(n r)=+1\right]$ domains are $\bar{U}$ and $\bar{V}$, respectively, [see Figure (1)]. Definitions of the $n l(n r)$ dimensional operators $\mathcal{D}_{l}\left(\mathcal{D}_{r}\right)$ are given by

$$
\mathcal{D}_{l}=\mathcal{P}_{l}^{-1} \mathcal{Q}_{l} ; \quad-1 \leq x \leq 0 \quad ; \quad \mathcal{D}_{r}=\mathcal{P}_{r}^{-1} \mathcal{Q}_{r} ; \quad 0 \leq x \leq 1
$$

and $\mathbf{e}_{\mathbf{i}_{-}}$and $\mathbf{e}_{\mathbf{i}_{+}}$are given by $\mathbf{e}_{\mathbf{i}_{-}}=[0,0, \ldots, 0,1]^{T}$ and $\mathbf{e}_{\mathbf{i}_{+}}=[1,0, \ldots, 0,0]^{T}$. Note that any two SBP operators $\mathcal{D}_{l}$, and $\mathcal{D}_{r}$ would suffice in equation (11). In fact, grid discontinuities $\left(\Delta x_{l} \neq \Delta x_{r}\right)$, nonuniform grids, or even operators of different order or type could be used. ${ }^{3}$

What remains is to determine the values of the interface parameters $l_{00}, \cdots, l_{p p}, r_{00}, \cdots r_{p p}$ that lead to stability, conservation and accuracy.

\subsubsection{Stability}

To facilitate the derivation of precise stability bounds for the interface parameters, we confine our discussion to the cases $p \leq 2$; i.e. only the solution and the first two derivatives are penalized at the interface. In this case, equation (11) simplifies to

$$
\begin{aligned}
\mathcal{P}_{l} \mathbf{U}_{t}+a \mathcal{P}_{l} \mathcal{D}_{l} \mathbf{U} & =\epsilon \mathcal{P}_{l} \mathcal{D}_{l} \mathcal{D}_{l} \mathbf{U} \\
& +\left\{l_{00}\left[U_{i}-V_{i}\right]+l_{01}\left[\left(\mathcal{D}_{l}^{1} U\right)_{i}-\left(\mathcal{D}_{r}^{1} V\right)_{i}\right]+l_{02}\left[\left(\mathcal{D}_{l}^{2} U\right)_{i}-\left(\mathcal{D}_{r}^{2} V\right)_{i}\right]\right\}\left[\mathcal{I}_{l}\right]^{T} \mathbf{e}_{\mathbf{i}_{-}} \\
& +\left\{l_{10}\left[U_{i}-V_{i}\right]+l_{11}\left[\left(\mathcal{D}_{l}^{1} U\right)_{i}-\left(\mathcal{D}_{r}^{1} V\right)_{i}\right]+l_{12}\left[\left(\mathcal{D}_{l}^{2} U\right)_{i}-\left(\mathcal{D}_{r}^{2} V\right)_{i}\right]\right\}\left[\mathcal{D}_{l}\right]^{T} \mathbf{e}_{\mathbf{i}_{-}} \\
& +\left\{l_{20}\left[U_{i}-V_{i}\right]+l_{21}\left[\left(\mathcal{D}_{l}^{1} U\right)_{i}-\left(\mathcal{D}_{r}^{1} V\right)_{i}\right]+l_{22}\left[\left(\mathcal{D}_{l}^{2} U\right)_{i}-\left(\mathcal{D}_{r}^{2} V\right)_{i}\right]\right\}\left[\mathcal{D}_{l}^{2}\right]^{T} \mathbf{e}_{\mathbf{i}_{-}} \\
\mathcal{P}_{r} \mathbf{V}_{t}+a \mathcal{P}_{r} \mathcal{D}_{r} \mathbf{V} & =\epsilon \mathcal{P}_{r} \mathcal{D}_{r} \mathcal{D}_{r} \mathbf{V} \\
& +\left\{r_{00}\left[V_{i}-U_{i}\right]+r_{01}\left[\left(\mathcal{D}_{r}^{1} V\right)_{i}-\left(\mathcal{D}_{l}^{1} U\right)_{i}\right]+r_{02}\left[\left(\mathcal{D}_{r}^{2} V\right)_{i}-\left(\mathcal{D}_{l}^{2} U\right)_{i}\right]\right\}\left[\mathcal{I}_{r}\right]^{T} \mathbf{e}_{\mathbf{i}_{+}} \\
& +\left\{r_{10}\left[V_{i}-U_{i}\right]+r_{11}\left[\left(\mathcal{D}_{r}^{1} V\right)_{i}-\left(\mathcal{D}_{l}^{1} U\right)_{i}\right]+r_{12}\left[\left(\mathcal{D}_{r}^{2} V\right)_{i}-\left(\mathcal{D}_{l}^{2} U\right)_{i}\right]\right\}\left[\mathcal{D}_{r}\right]^{T} \mathbf{e}_{\mathbf{i}_{+}} \\
& +\left\{r_{20}\left[V_{i}-U_{i}\right]+r_{21}\left[\left(\mathcal{D}_{r}^{1} V\right)_{i}-\left(\mathcal{D}_{l}^{1} U\right)_{i}\right]+r_{22}\left[\left(\mathcal{D}_{r}^{2} V\right)_{i}-\left(\mathcal{D}_{l}^{2} U\right)_{i}\right]\right\}\left[\mathcal{D}_{r}^{2}\right]^{T} \mathbf{e}_{\mathbf{i}_{+}}
\end{aligned}
$$

The values of the interface parameters $l_{00}, l_{01}, l_{02}, l_{10}, l_{11}, l_{12}, l_{20}, l_{21}, l_{22}$, and $r_{00}, r_{01}, r_{02}, r_{10}, r_{11}, r_{12}, r_{20}, r_{21}, r_{22}$, that result in a stable interface are given in the following theorem.

Theorem 1 The approximation (12) of the problem (9) is stable if the eighteen parameters are related by the six equalities

$$
\begin{aligned}
l_{00}=r_{00}+a & ; \quad l_{01}=r_{01}-\epsilon \\
r_{02}=l_{02} \quad ; \quad r_{20}=l_{20} & ; \quad r_{21}=l_{21} ; \quad r_{22}=l_{22}
\end{aligned}
$$

and constrained by the following inequalities

$$
\begin{gathered}
2\left[r_{11}+l_{11}\right] \leq[(\alpha+\beta) \epsilon] \\
\frac{\left[\epsilon(-\alpha+\beta)-\left(r_{11}-l_{11}\right)\right]^{2}}{\epsilon(\alpha+\beta)-2\left(r_{11}+l_{11}\right)} \leq[(\alpha+\beta) \epsilon] \\
\left.l_{22} \leq-\frac{\left(+2 r_{21}+r_{12}+l_{12}\right)^{2}}{4\left[\epsilon(\alpha+\beta)-2\left(r_{11}+l_{11}\right)\right]}-\frac{\left\{\left(l_{12}-r_{12}\right)+\frac{\left[\epsilon(-\alpha+\beta)-\left(r_{11}-l_{11}\right)\right]\left(+2 r_{21}+r_{12}+l_{12}\right)}{\left[\epsilon(\alpha+\beta)-2\left(r_{11}+l_{11}\right)\right]}\right.}{4\left\{[(\alpha+\beta) \epsilon]-\frac{\left[\epsilon(-\alpha+\beta)-\left(r_{11}-l_{11}\right)\right]^{2}}{\left[\epsilon(\alpha+\beta)-2\left(r_{11}+l_{11}\right)\right]}\right.}\right\}^{2}
\end{gathered}
$$

\footnotetext{
${ }^{3}$ Even material interfaces between adjoining media are allowed (see Nordströ and Gustafsson [27]).
} 


$$
\begin{aligned}
l_{00} \leq & \frac{a}{2}-\frac{\left[t_{1}+t_{2}\right]^{2}}{8[(\alpha+\beta) \epsilon]\left[s_{1}+s_{2}\right]}-\frac{\left[t_{1}-t_{2}+\frac{\left[s_{1}-s_{2}\right]\left[t_{1}+t_{2}\right]}{2\left(s_{1}+s_{2}\right)}\right]^{2}}{4(\alpha+\beta) \epsilon\left[1-\frac{\left[s_{1}-s_{2}\right]^{2}}{2\left[s_{1}+s_{2}\right]}\right]} \\
- & \frac{\left\{2 x_{1}+\frac{\left(t_{1}+t_{2}\right)\left(y_{1}+y_{2}\right)}{2(\alpha+\beta) \epsilon\left(s_{1}+s_{2}\right)}+\frac{\left[t_{1}-t_{2}+\frac{\left(s_{1}-s_{2}\right)\left(t_{1}+t_{2}\right)}{2\left(s_{1}+s_{2}\right)}\right]\left[y_{1}-y_{2}+\frac{\left(s_{1}-s_{2}\right)\left(y_{1}+y_{2}\right)}{2\left(s_{1}+s_{2}\right)}\right]^{2}}{4(\alpha+\beta) \epsilon\left[1-\frac{\left[s_{1}-s_{2}\right]^{2}}{2\left[s_{1}+s_{2}\right]}\right]}\right\}^{2}}{-4\left\{l_{22}+\frac{\left[y_{1}+y_{2}\right]^{2}}{8[(\alpha+\beta) \epsilon]\left[s_{1}+s_{2}\right]}+\frac{\left[y_{1}-y_{2}+\frac{\left[s_{1}-s_{2}\right]\left[y_{1}+y_{2}\right]}{2\left(s_{1}+s_{2}\right)}\right]^{2}}{4(\alpha+\beta) \epsilon\left[1-\frac{\left[s_{1}-s_{2}\right]^{2}}{2\left[s_{1}+s_{2}\right]}\right]}\right\}}
\end{aligned}
$$

with

$$
\begin{aligned}
t_{1}=\left(\epsilon+l_{01}+l_{10}\right) & ; \quad t_{2}=\left(l_{01}+r_{10}\right) \\
r_{11}=\epsilon\left[(-\alpha+3 \beta) / 4-s_{1}(\alpha+\beta)\right] & ; \quad l_{11}=\epsilon\left[(3 \alpha-\beta) / 4-s_{2}(\alpha+\beta)\right], \\
x_{1}=\left(r_{20}+l_{02}\right) & ; \quad y_{1}=\left(l_{12}+r_{21}\right) ; y_{2}=\left(r_{12}+r_{21}\right)
\end{aligned}
$$

and

$$
\alpha=\frac{1}{\mathbf{e}_{\mathbf{i}_{-}}{ }^{T} \mathcal{P}_{l}^{-1} \mathbf{e}_{\mathbf{i}_{-}}} \quad ; \quad \beta=\frac{1}{\mathbf{e}_{\mathbf{i}_{+}}{ }^{T} \mathcal{P}_{r}^{-1} \mathbf{e}_{\mathbf{i}_{+}}}
$$

Proof: Stability of (12) follows if the interface treatment at $x=x_{i}$ is of a dissipative nature. The energy method applied to equation (12) (multiplying the discrete equations in the left and right subdomains by $\mathbf{U}^{T}$ and $\mathbf{V}^{T}$ respectively, and adding the result to its transpose), and using the relation $Q+Q^{T}=B$ from the SBP rule (6), yields

$$
\begin{array}{rlcc}
\frac{d}{d t}\left[\|\mathbf{U}\|_{\mathcal{P}_{l}}^{2}+\|\mathbf{V}\|_{\mathcal{P}_{r}}^{2}\right] & & \\
+2 \epsilon\left[\left\|\mathcal{D}_{l} \mathbf{U}\right\|_{\mathcal{P}_{l}}^{2}+\left\|\mathcal{D}_{r} \mathbf{V}\right\|_{\mathcal{P}_{r}}^{2}\right] & = & {\left[2 \epsilon U \mathcal{D}_{l} U-a U^{2}\right]^{i_{-}}+\left[2 \epsilon V \mathcal{D}_{r} V-a V^{2}\right]_{i_{+}}} \\
& + & 2 & U_{i}\left\{l_{00}\left[U_{i}-V_{i}\right]+l_{01}\left[\left(\mathcal{D}_{l} U\right)_{i}-\left(\mathcal{D}_{r} V\right)_{i}\right]+l_{02}\left[\left(\mathcal{D}_{l}^{2} U\right)_{i}-\left(\mathcal{D}_{r}^{2} V\right)_{i}\right]\right\} \\
& + & 2\left(\mathcal{D}_{l}^{1} U\right)_{i}\left\{l_{10}\left[U_{i}-V_{i}\right]+l_{11}\left[\left(\mathcal{D}_{l} U\right)_{i}-\left(\mathcal{D}_{r} V\right)_{i}\right]+l_{12}\left[\left(\mathcal{D}_{l}^{2} U\right)_{i}-\left(\mathcal{D}_{r}^{2} V\right)_{i}\right]\right\} \\
& + & 2\left(\mathcal{D}_{l}^{2} U\right)_{i}\left\{l_{20}\left[U_{i}-V_{i}\right]+l_{21}\left[\left(\mathcal{D}_{l} U\right)_{i}-\left(\mathcal{D}_{r} V\right)_{i}\right]+l_{22}\left[\left(\mathcal{D}_{l}^{2} U\right)_{i}-\left(\mathcal{D}_{r}^{2} V\right)_{i}\right]\right\} \\
& + & 2 \quad V_{i}\left\{r_{00}\left[V_{i}-U_{i}\right]+r_{01}\left[\left(\mathcal{D}_{r} V\right)_{i}-\left(\mathcal{D}_{l} U\right)_{i}\right]+r_{02}\left[\left(\mathcal{D}_{r}^{2} V\right)_{i}-\left(\mathcal{D}_{l}^{2} U\right)_{i}\right]\right\} \\
& +2\left(\mathcal{D}_{r}^{1} V\right)_{i}\left\{r_{10}\left[V_{i}-U_{i}\right]+r_{11}\left[\left(\mathcal{D}_{r} V\right)_{i}-\left(\mathcal{D}_{l} U\right)_{i}\right]+r_{12}\left[\left(\mathcal{D}_{r}^{2} V\right)_{i}-\left(\mathcal{D}_{l}^{2} U\right)_{i}\right]\right\} \\
& +2\left(\mathcal{D}_{r}^{2} V\right)_{i}\left\{r_{20}\left[V_{i}-U_{i}\right]+r_{21}\left[\left(\mathcal{D}_{r} V\right)_{i}-(\mathcal{D} l U)_{i}\right]+r_{22}\left[\left(\mathcal{D}_{r}^{2} V\right)_{i}-\left(\mathcal{D}_{l}^{2} U\right)_{i}\right]\right\}
\end{array}
$$

Note again that we have neglected the outer boundary terms.

A small portion of the diffusion terms $\left\|\mathcal{D}_{l} \mathbf{U}\right\|_{\mathcal{P}_{l}}$ and $\left\|\mathcal{D}_{r} \mathbf{V}\right\|_{\mathcal{P}_{r}}$ arising at the interface can be moved to the (RHS) to help in the proof of stability. Defining

$$
\overline{\mathcal{P}}_{l}=\mathcal{P}_{l}-\alpha \mathbf{e}_{\mathbf{i}_{-}} \mathbf{e}_{\mathbf{i}_{-}}{ }^{T} \quad ; \quad \overline{\mathcal{P}}_{r}=\mathcal{P}_{r}-\beta \mathbf{e}_{\mathbf{i}_{+}} \mathbf{e}_{\mathbf{i}_{+}}{ }^{T}
$$

with $\alpha$ and $\beta$ as defined in equation (19) and collecting all the interface terms, equation (20) becomes

$$
\frac{d}{d t}\left[\|\mathbf{U}\|_{\mathcal{P}_{l}}^{2}+\|\mathbf{V}\|_{\mathcal{P}_{r}}^{2}\right]+2 \epsilon\left[\left\|\mathcal{D}_{l} \mathbf{U}\right\|_{\overline{\mathcal{P}}_{l}}^{2}+\left\|\mathcal{D}_{r} \mathbf{V}\right\|_{\overline{\mathcal{P}}_{r}}^{2}\right]=\bar{\Upsilon}_{i}
$$

with the interface term $\bar{\Upsilon}_{i}=\left[\mathcal{J}_{i}\right]^{T} \bar{M}_{i} \mathcal{J}_{i}$ defined by

$$
\begin{aligned}
& \mathcal{J}_{i}=\left[U_{i}, V_{i},\left(\mathcal{D}_{l} U\right)_{i},\left(\mathcal{D}_{r} V\right)_{i},\left(\mathcal{D}_{l}^{2} U\right)_{i},\left(\mathcal{D}_{r}^{2} V\right)_{i}\right]^{T} \\
& \bar{M}_{i}=\left[\begin{array}{rccccc}
\left(-a+2 l_{00}\right) & -\left(l_{00}+r_{00}\right) & \left(\epsilon+l_{01}+l_{10}\right) & -\left(l_{01}+r_{10}\right) & \left(l_{02}+l_{20}\right) & -\left(l_{02}+r_{20}\right) \\
-\left(l_{00}+r_{00}\right) & \left(+a+2 r_{00}\right) & -\left(r_{01}+l_{10}\right) & \left(-\epsilon+r_{01}+r_{10}\right) & -\left(l_{20}+r_{02}\right) & \left(r_{02}+r_{20}\right) \\
\left(\epsilon+l_{01}+l_{10}\right) & -\left(r_{01}+l_{10}\right) & 2\left(l_{11}-\alpha \epsilon\right) & -\left(l_{11}+r_{11}\right) & \left(l_{12}+l_{21}\right) & -\left(l_{12}+r_{21}\right) \\
-\left(l_{01}+r_{10}\right) & \left(-\epsilon+r_{01}+r_{10}\right) & -\left(l_{11}+r_{11}\right) & 2\left(r_{11}-\beta \epsilon\right) & -\left(l_{21}+r_{12}\right) & \left(r_{21}+r_{12}\right) \\
\left(l_{02}+l_{20}\right) & -\left(l_{20}+r_{02}\right) & \left(l_{12}+l_{21}\right) & -\left(l_{21}+r_{12}\right) & 2 l_{22} & -\left(l_{22}+r_{22}\right) \\
-\left(l_{02}+r_{20}\right) & \left(r_{02}+r_{20}\right) & -\left(l_{12}+r_{21}\right) & \left(r_{21}+r_{12}\right) & -\left(l_{22}+r_{22}\right) & 2 r_{22}
\end{array}\right]
\end{aligned}
$$

The origins of equation (19) are derived in reference [9] including a proof of the definiteness of $\overline{\mathcal{P}}_{l}$ and $\overline{\mathcal{P}}_{r}$. 
Stability of the interface is guaranteed if the matrix $\bar{M}_{i}$ is negative semi-definite; i.e. $\bar{\Upsilon}_{i}=\left[\mathcal{J}_{i}\right]^{T} \bar{M}_{i} \mathcal{J}_{i} \leq 0$. The definiteness of a symmetric matrix is governed by the sign of its eigenvalues. Thus, Sylvester's Theorem is used to systematically rotate $\bar{M}_{i}$ into diagonal form while maintaining the signs of its eigenvalues.

The first rotation of $\bar{M}_{i}$ is the following similarity transformation. Define the new vector $\hat{\mathcal{J}}_{i}=\mathcal{S} \mathcal{J}_{i}$ such that:

$$
\hat{\mathcal{J}}_{i}=\frac{1}{\sqrt{2}}\left[\begin{array}{c}
U_{i}+V_{i} \\
U_{i}-V_{i} \\
\left(\mathcal{D}_{l} U\right)_{i}+\left(\mathcal{D}_{r} V\right)_{i} \\
\left(\mathcal{D}_{l} U\right)_{i}-\left(\mathcal{D}_{r} V\right)_{i} \\
\left(\mathcal{D}_{l}^{2} U\right)_{i}+\left(\mathcal{D}_{r}^{2} V\right)_{i} \\
\left(\mathcal{D}_{l}^{2} U\right)_{i}-\left(\mathcal{D}_{r}^{2} V\right)_{i}
\end{array}\right]=\frac{1}{\sqrt{2}}\left[\begin{array}{cccccc}
1 & 1 & 0 & 0 & 0 & 0 \\
1 & -1 & 0 & 0 & 0 & 0 \\
0 & 0 & 1 & 1 & 0 & 0 \\
0 & 0 & 1 & -1 & 0 & 0 \\
0 & 0 & 0 & 0 & 1 & 1 \\
0 & 0 & 0 & 0 & 1 & -1
\end{array}\right]\left[\begin{array}{c}
U_{i} \\
V_{i} \\
\left(\mathcal{D}_{l} U\right)_{i} \\
\left(\mathcal{D}_{r} V\right)_{i} \\
\left(\mathcal{D}_{l}^{2} U\right)_{i} \\
\left(\mathcal{D}_{r}^{2} V\right)_{i}
\end{array}\right]
$$

The rotation matrix $\mathcal{S}$ replaces the stability condition given in equation (24) with the following equivalent condition:

$$
\mathcal{J}_{i}^{T} M^{i} \mathcal{J}_{i}=\mathcal{J}_{i}^{T} \mathcal{S}^{T} \mathcal{S} \bar{M}_{i} \mathcal{S}^{T} \mathcal{S} \mathcal{J}_{i}=\hat{\mathcal{J}}_{i}^{T} \hat{M}_{i} \hat{\mathcal{J}}_{i} \leq 0 \quad
$$

The rotated matrix $\hat{M}_{i}$ has zeros on the main diagonal. (See reference [9] for details.) Thus, definiteness of $\hat{M}_{i}$ requires all off-diagonal terms in the respective rows be zero, and produces the generalized conservation conditions

$$
\begin{aligned}
l_{00}=r_{00}+a & ; \quad l_{01}=r_{01}-\epsilon \\
r_{02}=l_{02} \quad ; \quad r_{20}=l_{20} & ; \quad r_{21}=l_{21} ; \quad r_{22}=l_{22}
\end{aligned}
$$

The necessary algebra is greatly simplified through a change of variables. Defining

$$
\begin{array}{rll}
t_{1}=\left(\epsilon+l_{01}+l_{10}\right) & ; \quad t_{2}=\left(l_{01}+r_{10}\right) \\
r_{11}=\epsilon\left[(-\alpha+3 \beta) / 4-s_{1}(\alpha+\beta)\right] & ; \quad l_{11}=\epsilon\left[(3 \alpha-\beta) / 4-s_{2}(\alpha+\beta)\right], \\
x_{1}=\left(r_{20}+l_{02}\right) & ; & y_{1}=\left(l_{12}+r_{21}\right) ; y_{2}=\left(r_{12}+r_{21}\right)
\end{array}
$$

yields the reduced matrix

$$
\bar{M}_{i}=\left[\begin{array}{ccccll}
0 & 0 & 0 & 0 & 0 & 0 \\
0 & -2 a+4 l_{00} & t_{1}-t_{2} & t_{1}+t_{2} & 0 & 2 x_{1} \\
0 & t_{1}-t_{2} & -((\alpha+\beta) \epsilon) & (\alpha+\beta) \epsilon(s 1-s 2) & 0 & y_{1}-y_{2} \\
0 & t_{1}+t_{2} & (\alpha+\beta) \epsilon(s 1-s 2) & -2(\alpha+\beta) \epsilon(s 1+s 2) & 0 & y_{1}+y_{2} \\
0 & 0 & 0 & 0 & 0 & 0 \\
0 & 2 x_{1} & y_{1}-y_{2} & y_{1}+y_{2} & 0 & 4 l_{22}
\end{array}\right]
$$

Repeated application of Sylvester's Theorem produces the stability inequalities

$$
\begin{aligned}
0 & \leq\left[s_{1}+s_{2}\right] \\
0 & \leq 1-\frac{\left[s_{1}-s_{2}\right]^{2}}{2\left[s_{1}+s_{2}\right]} \\
l_{22} & \leq-\frac{\left[y_{1}+y_{2}\right]^{2}}{8[(\alpha+\beta) \epsilon]\left[s_{1}+s_{2}\right]}-\frac{\left[y_{1}-y_{2}+\frac{\left[s_{1}-s_{2}\right]\left[y_{1}+y_{2}\right]}{2\left(s_{1}+s_{2}\right)}\right]^{2}}{4(\alpha+\beta) \epsilon\left[1-\frac{\left[s_{1}-s_{2}\right]^{2}}{2\left[s_{1}+s_{2}\right]}\right]} \\
l_{00} & \leq \frac{a}{2}-\frac{\left[t_{1}+t_{2}\right]^{2}}{8[(\alpha+\beta) \epsilon]\left[s_{1}+s_{2}\right]}-\frac{\left[t_{1}-t_{2}+\frac{\left[s_{1}-s_{2}\right]\left[t_{1}+t_{2}\right]}{2\left(s_{1}+s_{2}\right)}\right]^{2}}{4(\alpha+\beta) \epsilon\left[1-\frac{\left[s_{1}-s_{2}\right]^{2}}{2\left[s_{1}+s_{2}\right]}\right]} \\
& -\frac{-42 x_{1}+\frac{\left(t_{1}+t_{2}\right)\left(y_{1}+y_{2}\right)}{2(\alpha+\beta) \epsilon\left(s_{1}+s_{2}\right)}+\frac{\left[t_{1}-t_{2}+\frac{\left(s_{1}-s_{2}\right)\left(t_{1}+t_{2}\right)}{2\left(s_{1}+s_{2}\right)}\right]\left[y_{1}-y_{2}+\frac{\left(s_{1}-s_{2}\right)\left(y_{1}+y_{2}\right)}{2\left(s_{1}+s_{2}\right)}\right]}{4(\alpha+\beta) \epsilon\left[1-\frac{\left[s_{1}-s_{2}\right]^{2}}{2\left[s_{2}+s_{2}\right]}\right]}}{2}{ }^{2} \\
& \left.-4 l_{22}+\frac{\left[y_{1}+y_{2}\right]^{2}}{\left.8[(\alpha+\beta) \epsilon] s_{1}+s_{2}\right]}+\frac{\left[y_{1}-y_{2}+\frac{\left[s_{1}-s_{2}\right]\left[y_{1}+y_{2}\right]}{2\left(s_{1}+s_{2}\right)}\right]^{2}}{4(\alpha+\beta) \epsilon\left[1-\frac{\left.s_{1}-s_{2}\right]^{2}}{2\left[s_{1}+s_{2}\right]}\right]^{2}}\right\}
\end{aligned}
$$

which are the desired result. 
Remark. As mentioned earlier we can easily extend this result to strong stability by including the outer boundary conditions.

Remark. A general interface procedure including derivative terms up to $p^{t h}$-order and satisfying an $L_{2}$ stability condition, would require similar inequalities for all interface parameters $l_{00} \rightarrow l_{p p}$, and $r_{00} \rightarrow r_{p p}$. Such inequalities could in principle be derived using a similar approach, but are not presented herein.

\subsubsection{Pointwise Stability}

Three distinct groups of terms appear in equation (22). The groups include the solution terms $\|\mathbf{U}\|_{\mathcal{P}_{l}}^{2}$ and $\|\mathbf{V}\|_{\mathcal{P}_{r}}^{2}$, the derivative terms $\left\|\mathcal{D}_{l} \mathbf{U}\right\|_{\overline{\mathcal{P}}_{l}}^{2}$ and $\left\|\mathcal{D}_{r} \mathbf{V}\right\|_{\overline{\mathcal{P}}_{r}}^{2}$, and the interface term $\Upsilon_{i}$. The solution and derivative terms are weakly bounded in $\mathcal{P}$-norms for finite times, while the interface term is locally pointwise bounded.

Note that equation (22) does not establish the pointwise stability of the solution at interior gridpoints. To establish interior pointwise boundedness, a discrete Sobolev inequality is required. A proof of the discrete Sobolev inequality,

$$
\left(U_{i}\right)^{2} \leq c_{1}\|\mathbf{U}\|_{\mathcal{P}}^{2}+c_{2}\|\mathcal{D} \mathbf{U}\|_{\mathcal{P}}^{2}
$$

derived specifically for single-domain, SBP operators, is presented in reference [9]. This inequality, combined with equation (22), provides the link between $\mathcal{P}$-boundedness of $\mathbf{U}$ and $\mathcal{D} \mathbf{U}$, and pointwise boundedness of the solution over the entire domain, and lead to the following theorem.

Theorem 2 For any grid function $\mathbf{U}$ on $\left(0 \leq x_{j} \leq l\right) ; j=0,1, \cdots, n$, and a consistent derivative operator $\mathcal{D}$ of rank $n-1$, then $\mathcal{P}$-boundedness of $\|\mathbf{U}\|_{\mathcal{P}}^{2}$ and $\|\mathcal{D} \mathbf{U}\|_{\mathcal{P}}^{2}$, implies a pointwise estimate of the form

$$
\|\mathbf{U}\|_{\infty}^{2} \leq\left(c_{m p m}+l^{-1}\right)\|\mathbf{U}\|^{2}+c_{m p m}^{-1}\|\mathcal{D} \mathbf{U}\|^{2}
$$

Proof : Refer to reference [21] for the proof of Theorem (2). Extension to two domains is straightforward.

Remark. In addition to providing a stronger measure of stability, a pointwise stability estimate can be a necessary condition used in proofs of global accuracy. Indeed, a pointwise estimate is the critical condition used in the work of Svärd and Nordström [34], and in Section 4 of this work, to establish a sharp estimate of global solution accuracy.

Remark. A consistent derivative operator $\mathcal{D}$ of rank $n-1$, is needed to extend the classical proofs[21], developed using first-order difference operators, to those using high-order differences.

\subsubsection{Primal Conservation}

We are interested in numerical solutions to the 1-D equation in divergence form

$$
u_{t}+f_{x}=0,|x| \leq 1, t \geq 0 ; f=a u-\epsilon u_{x}
$$

where $f$ involves convective and diffusive terms arising from for example the linear Burgers' equation. Consider the special case for which $f$ is discontinuous (i.e. $f_{x}$ is undefined). Then equation (31) must be replaced by the related weak statement

$$
\left.\int_{-1}^{1} \Phi(x, t) u(x)\right|_{0} ^{T} d x+\left.\int_{0}^{T} \Phi(x, t) f(t)\right|_{-1} ^{+1} d t=\int_{0}^{T} \int_{-1}^{1}\left[\Phi_{t}(x, t) u(x, t)+\Phi_{x}(x, t) f(u(x, t))\right] d x d t
$$

where $\Phi(x, t)$ is an arbitrary smooth function.

Semi-discrete operators must satisfy an additional constraint: conservation, if they are to accurately resolve discontinuous data. Discrete conservation can be defined in many ways, but herein it is defined as follows:

Definition 2 Assume that the discrete solution converges to a function $u(x, t)$. If this convergent limit function $u(x, t)$ is a weak solution to equation (31) that satisfies equation (32), then the semi-discretization is said to be "conservative".

In practical terms, semi-discretize operators used for equation (31) must allow for discrete manipulation to a form that is discretely equivalent to equation (32): i.e. spatial integrals replaced with discrete quadratures, and must converge 
to equation (32) in the limit of infinite resolution. As will be shown, this is a delicate process at internal boundaries between subdomains.

A variant of the classical Lax-Wendroff Theorem [22] is now presented in the context of the SBP formulation defined in equation (12). Special attention is given to the interface conditions, and the diffusive terms. For ease of presentation, we shall confine our attention to the case $p \leq 1$, i.e. only the solution and the first derivative are penalized across the interface.

Theorem 3 Two conditions are necessary if approximation (12) is to be conservative (in the $\mathcal{P}$ norm) across the interface. They are

$$
l_{00}=r_{00}+a, \quad l_{01}=r_{01}-\epsilon
$$

Proof : Multiplying (12) with $\boldsymbol{\Phi}_{l}^{T} \mathcal{P}_{l}$ and $\boldsymbol{\Phi}_{r}^{T} \mathcal{P}_{r}$, (neglecting the farfield boundary terms and assuming for clarity that $p \leq 1$ ) leads to

$$
\begin{aligned}
& \boldsymbol{\Phi}_{l}^{T} \mathcal{P}_{l} \mathbf{U}_{t}+\boldsymbol{\Phi}_{r}^{T} \mathcal{P}_{r} \mathbf{V}_{t} \\
& +a\left[\boldsymbol{\Phi}_{l}^{T} \mathcal{P}_{l} \mathcal{D}_{l} \mathbf{U}+\boldsymbol{\Phi}_{r}^{T} \mathcal{P}_{r} \mathcal{D}_{r} \mathbf{V}\right] \\
& -\epsilon\left[\boldsymbol{\Phi}_{l}^{T} \mathcal{P}_{l} \mathcal{D}_{l} \mathcal{D}_{l} \mathbf{U}+\boldsymbol{\Phi}_{r}^{T} \mathcal{P}_{r} \mathcal{D}_{r} \mathcal{D}_{r} \mathbf{V}\right]=l_{00} \Phi_{i}\left[U_{i}-V_{i}\right]+l_{01} \Phi_{i}\left[\left(\mathcal{D}_{l} U\right)_{i}-\left(\mathcal{D}_{r} V\right)_{i}\right] \\
& +\quad l_{10}\left(\Phi^{\prime}\right)_{i}\left[U_{i}-V_{i}\right]+l_{11}\left(\Phi^{\prime}\right)_{i}\left[\left(\mathcal{D}_{l} U\right)_{i}-\left(\mathcal{D}_{r} V\right)_{i}\right] \\
& +r_{00} \Phi_{i}\left[V_{i}-U_{i}\right]+r_{01} \Phi_{i}\left[\left(\mathcal{D}_{r} V\right)_{i}-\left(\mathcal{D}_{l} U\right)_{i}\right] \\
& +r_{10}\left(\Phi^{\prime}\right)_{i}\left[V_{i}-U_{i}\right]+r_{11}\left(\Phi^{\prime}\right)_{i}\left[\left(\mathcal{D}_{r} V\right)_{i}-\left(\mathcal{D}_{l} U\right)_{i}\right]
\end{aligned}
$$

where $\Phi_{i}$ and $\left(\Phi^{\prime}\right)_{i}$ are the values of the test function and its derivative at the interface location $x_{i}$. Both are sufficiently continuous across the interface.

Note the ambiguity in the meaning of conservation for the diffusive terms. For example, using the SBP rules $[(5)-(6)]$ once on the diffusion terms yields,

$$
\begin{aligned}
\boldsymbol{\Phi}_{l}^{T} \mathcal{P}_{l} \mathcal{D}_{l} \mathcal{D}_{l} \mathbf{U}+\boldsymbol{\Phi}_{r}^{T} \mathcal{P}_{r} \mathcal{D}_{r} \mathcal{D}_{r} \mathbf{V} & \left.=-\left(\Phi_{l}^{\prime}\right)^{T} \mathcal{P}_{l} \mathcal{D}_{l} U\right) \\
& \left.+\left.\left[\Phi_{l}\left(\mathcal{D}_{l} U\right)\right]\right|_{-1} ^{i_{-}}+\left(\Phi_{r}^{\prime}\right)^{T} \mathcal{P}_{r} \mathcal{D}_{r} V\right) \\
& +\left.\left[\Phi_{r}\left(\mathcal{D}_{r} U\right)\right]\right|_{i_{+}} ^{+1}
\end{aligned}
$$

Rearranging the time terms, and using the SBP rules $[(5)-(6)]$ on the convection terms in equation (34), then simplifying using equation (35) produces the conservation equation

$$
\begin{aligned}
\frac{d}{d t}\left(\boldsymbol{\Phi}_{l}^{T} \mathcal{P}_{l} \mathbf{U}+\boldsymbol{\Phi}_{r}^{T} \mathcal{P}_{r} \mathbf{V}\right) & \\
+\left.\left[\Phi_{l}\left(a-\epsilon \mathcal{D}_{l}\right) U\right]\right|_{-1}+\left.\left[\Phi_{r}\left(a-\epsilon \mathcal{D}_{r}\right) V\right]\right|^{+1} & =\left[\left(\Phi_{l}\right)_{t}^{T} \mathcal{P}_{l} \mathbf{U}+\left(\Phi_{r}\right)_{t}^{T} \mathcal{P}_{r} \mathbf{V}\right] \\
& +\left(\Phi^{\prime}{ }_{l}^{T} \mathcal{P}_{l}\left(a \mathcal{I}_{l}-\epsilon \mathcal{D}_{l}\right) \mathbf{U}+\left(\Phi^{\prime}{ }_{r}\right)^{T} \mathcal{P}_{r}\left(a \mathcal{I}_{r}-\epsilon \mathcal{D}_{r}\right) \mathbf{V}\right. \\
& +\Phi_{i}\left(l_{00}-r_{00}-a\right)\left(U_{i}-V_{i}\right) \\
& +\Phi_{i}\left(l_{01}-r_{01}+\epsilon\right)\left(\mathcal{D} U_{i}-\mathcal{D} V_{i}\right) \\
& +\left(\Phi^{\prime}\right)_{i}\left(l_{10}-r_{10}\right)\left(U_{i}-V_{i}\right) \\
& +\left(\Phi^{\prime}\right)_{i}\left(l_{11}-r_{11}\right)\left(\mathcal{D} U_{i}-\mathcal{D} V_{i}\right)
\end{aligned}
$$

Integrating equation (36) with respect to time, applying the conservation conditions given by equation (33) and the additional conditions $l_{10}=r_{10}$ and $l_{11}=r_{11}$ produces the equation

$$
\begin{aligned}
\left.\left(\boldsymbol{\Phi}_{l}^{T} \mathcal{P}_{l} \mathbf{U}+\boldsymbol{\Phi}_{r}^{T} \mathcal{P}_{r} \mathbf{V}\right)\right|_{0} ^{T} & \\
+\int_{0}^{T}\left\{\left.\left[\Phi_{l}\left(a-\epsilon \mathcal{D}_{l}\right) U\right]\right|_{-1}+\left.\left[\Phi_{r}\left(a-\epsilon \mathcal{D}_{r}\right) V\right]\right|^{+1}\right\} d t & =\int_{0}^{T}\left[\left(\Phi_{l}\right)_{t}^{T} \mathcal{P}_{l} \mathbf{U}+\left(\Phi_{r}\right)_{t}^{T} \mathcal{P}_{r} \mathbf{V}\right] d t \\
& +\int_{0}^{T}\left\{\left(\Phi^{\prime}{ }_{l}\right)^{T} \mathcal{P}_{l}\left(a \mathcal{I}_{l}-\epsilon \mathcal{D}_{l}\right) \mathbf{U}+\left(\Phi^{\prime}{ }_{r}\right)^{T} \mathcal{P}_{r}\left(a \mathcal{I}_{r}-\epsilon \mathcal{D}_{r}\right) \mathbf{V}\right\} d t
\end{aligned}
$$

which matches the weak solution given by equation (32) term by term, in the limit of infinite spatial resolution.

Using the SBP rules $[(5)-(6)]$ twice on the diffusion terms yields,

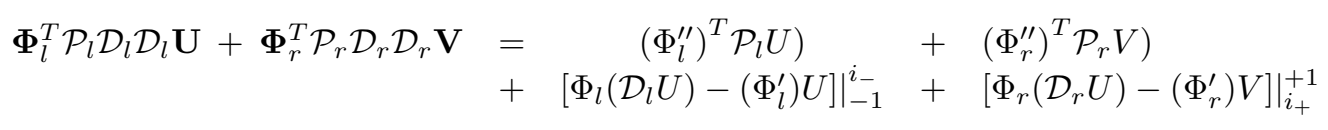


which leads to the resulting conservation equation

$$
\begin{aligned}
\frac{d}{d t}\left(\boldsymbol{\Phi}_{l}^{T} \mathcal{P}_{l} \mathbf{U}+\boldsymbol{\Phi}_{r}^{T} \mathcal{P}_{r} \mathbf{V}\right) & \\
+\left.\left[\Phi_{l}\left(a-\epsilon \mathcal{D}_{l}\right) U\right]\right|_{-1}+\left.\left[\Phi_{r}\left(a-\epsilon \mathcal{D}_{r}\right) V\right]\right|^{+1} & =\left[\left(\Phi_{l}\right)_{t}^{T} \mathcal{P}_{l} \mathbf{U}+\left(\Phi_{r}\right)_{t}^{T} \mathcal{P}_{r} \mathbf{V}\right] \\
& +\left(\Phi^{\prime}\right)^{T} \mathcal{P}_{l}\left(a \mathcal{I}_{l}\right) \mathbf{U}+\left(\Phi^{\prime}{ }_{r}\right)^{T} \mathcal{P}_{r}\left(a \mathcal{I}_{r}\right) \mathbf{V} \\
& +\epsilon\left(\Phi^{\prime \prime}{ }^{T}\right)^{T} \mathcal{P}_{l} \mathbf{U}+\epsilon\left(\Phi^{\prime \prime}{ }_{r}\right)^{T} \mathcal{P}_{r} \mathbf{V}-\epsilon\left[\left.\Phi_{l}^{\prime} U\right|_{-1}+\left.\Phi_{r}^{\prime} V\right|^{+1}\right] \\
& +\Phi_{i}\left(l_{00}-r_{00}-a\right)\left(U_{i}-V_{i}\right) \\
& +\Phi_{i}\left(l_{01}-r_{01}+\epsilon\right)\left(\mathcal{D} U_{i}-\mathcal{D} V_{i}\right) \\
& +\left(\Phi^{\prime}\right)_{i}\left(l_{10}-r_{10}-\epsilon\right)\left(U_{i}-V_{i}\right) \\
& +\left(\Phi^{\prime}\right)_{i}\left(l_{11}-r_{11}\right)\left(\mathcal{D} U_{i}-\mathcal{D} V_{i}\right)
\end{aligned}
$$

Integrating equation (39) with respect to time, applying the conservation conditions given by equation (33) and the additional conditions $l_{10}=r_{10}+\epsilon$ and $l_{11}=r_{11}$, yields an expression similar to equation (37) (see reference [9]) that matches the weak form of the advection diffusion equation in the limit of infinite spatial resolution.

Remark. Note that the diffusive constraint conditions for the variable $\Phi^{\prime}$ in equations (36) and (39) depend on the choice of definition for conservation. Still another definition (perhaps a linear combination of the two) would yield a

third set of constraints. This arbitrariness probably means that diffusive conservation is not as important as convective conservation.

\subsection{A Flux-Form Interface Penalty}

An SBP interface penalty (restricted for clarity to $p \leq 1$ ) that approximates equation $(9)$, motivated by the Local Discontinuous Galerkin (LDG) FEM proposed by Cockburn and Shu [11] is

$$
\begin{aligned}
& \mathcal{P}_{l} \mathbf{U}_{t}+a \mathcal{P}_{l} \mathcal{D}_{l} \mathbf{U}-\epsilon \mathcal{P}_{l} \mathcal{D}_{l} \phi=L_{00}\left[U_{i}-V_{i}\right] \quad \mathbf{e}_{\mathbf{i}_{-}}+L_{01}\left[(\phi)_{i}-(\psi)_{i}\right] \quad \mathbf{e}_{\mathbf{i}_{-}} \\
& \epsilon \mathcal{P}_{l}\left(\phi-\mathcal{D}_{l} \mathbf{U}\right) \quad=L_{10}\left[U_{i}-V_{i}\right] \quad \mathbf{e}_{\mathbf{i}_{-}}+L_{01}\left[(\phi)_{i}-(\psi)_{i}\right] \mathbf{e}_{\mathbf{i}_{-}} \\
& \mathbf{U}(x, 0) \quad=\quad 0 \text {. } \\
& \mathcal{P}_{r} \mathbf{V}_{t}+a \mathcal{P}_{r} \mathcal{D}_{r} \mathbf{V}-\epsilon \mathcal{P}_{r} \mathcal{D}_{r} \psi=R_{00}\left[V_{i}-U_{i}\right] \quad \mathbf{e}_{\mathbf{i}_{+}}+R_{01}\left[(\psi)_{i}-(\phi)_{i}\right] \quad \mathbf{e}_{\mathbf{i}_{+}} \\
& \epsilon \mathcal{P}_{r}\left(\psi-\mathcal{D}_{r} \mathbf{V}\right) \quad=R_{10}\left[V_{i}-U_{i}\right] \quad \mathbf{e}_{\mathbf{i}_{+}}+R_{11}\left[(\psi)_{i}-(\phi)_{i}\right] \quad \mathbf{e}_{\mathbf{i}_{+}} \\
& \mathbf{V}(x, 0) \quad=\quad \mathbf{0} \text {. }
\end{aligned}
$$

Theorem 4 The approximation (40) of the problem (9) is stable if the eight parameters are related by the two equalities

$$
L_{00}=R_{00}+a \quad ; \quad L_{01}=R_{01}-\epsilon
$$

and constrained by the following inequalities

$$
\begin{gathered}
2\left[R_{11}+L_{11}\right] \leq[(\alpha+\beta) \epsilon] \\
\frac{\left[\epsilon(-\alpha+\beta)-\left(R_{11}-L_{11}\right)\right]^{2}}{\epsilon(\alpha+\beta)-2\left(R_{11}+L_{11}\right)} \leq[(\alpha+\beta) \epsilon] \\
L_{00} \leq \frac{a}{2}-\frac{\left(\epsilon+2 L_{01}+L_{10}+R_{10}\right)^{2}}{4\left[\epsilon(\alpha+\beta)-2\left(R_{11}+L_{11}\right)\right]}-\frac{\left\{\epsilon+L_{10}-R_{10}+\frac{\left[\epsilon(-\alpha+\beta)-\left(R_{11}-L_{11}\right)\right]\left(\epsilon+2 L_{01}+L_{10}+R_{10}\right)}{\left[\epsilon(\alpha+\beta)-2\left(R_{11}+L_{11}\right)\right\}^{2}}\right.}{4\left\{(\alpha+\beta) \epsilon-\frac{\left[\epsilon(-\alpha+\beta)-\left(R_{11}-L_{11}\right)\right]^{2}}{\left.\epsilon(\alpha+\beta)-2\left(R_{11}+L_{11}\right)\right]}\right\}}
\end{gathered}
$$

where (as with the primal form)

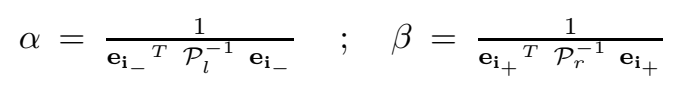

Proof: The stability proof for the LDG scheme is omitted, as it is equivalent to that used for the primal scheme. 
Remark. Although the stability boundaries for the eight interface parameters are identical for the primal and LDG schemes, as are the norms used in both cases, the two methods are stable in different combinations of terms: $\mathcal{D} U$ vs. $\phi$.

Remark: A general primal interface penalty that includes derivatives up to $p^{\text {th }}$-order, would require constraints on the interface parameters $L_{00} \rightarrow L_{p p}$, and $R_{00} \rightarrow R_{p p}$. A stability analysis could be used to identify conservation conditions, as well as the parameter space that guarantees $L_{2}$ stability.

Interior pointwise boundedness is established using a discrete Sobolev inequality. This inequality, (see reference [9] or [21]) combined with theorem (4), provides the link between $\mathcal{P}$-boundedness of $\mathbf{U}$ and $\phi$, and pointwise boundedness of the solution over the entire domain, and lead to the following theorem.

Theorem 5 For any grid function $\mathbf{U}$ on $\left(0 \leq x_{j} \leq l\right) ; j=0,1, \cdots, n$, a consistent derivative operator $\mathcal{D}$ of rank $n-1$, and $\|\phi\|^{2}$ as defined in equation (40), then $\mathcal{P}$-boundedness of $\|\mathbf{U}\|_{\mathcal{P}}^{2}$ and $\|\phi\|_{\mathcal{P}}^{2}$, implies a pointwise estimate of the form

$$
\|\mathbf{U}\|_{\infty}^{2} \leq\left(c_{m p m}+l^{-1}\right)\|\mathbf{U}\|^{2}+c_{m p m}{ }^{-1}\|\phi\|^{2}
$$

Proof : Refer to reference [21] for a proof of Theorem (5).

\subsection{Relating the Primal and Flux Formulations}

One might imagine that the primal and flux formulations are equivalent (as shown in FEM by Arnold et al $[2,3]$ ) with an appropriate change of interface variables. We begin by showing that the LDG formulation can be implemented in terms of the primal scheme if specific values of the interface parameters are used. For ease of presentation, we assume that only the solution and first derivatives are penalized across the interface.

Theorem 6 The LDG interface parameters $L_{00}-R_{11}$ are related to the primal interface parameters $l_{00}-r_{11}$ by the following relations:

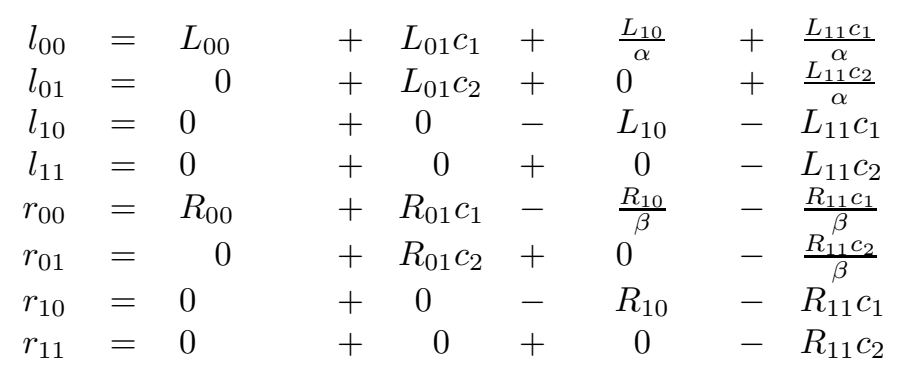

with

$$
c_{1}=\frac{\frac{1}{\epsilon}\left(\frac{L_{10}}{\alpha}+\frac{R_{10}}{\beta}\right)}{1-\frac{1}{\epsilon}\left(\frac{L_{11}}{\alpha}+\frac{R_{11}}{\beta}\right)} \quad ; \quad c_{2}=\frac{1}{1-\frac{1}{\epsilon}\left(\frac{L_{11}}{\alpha}+\frac{R_{11}}{\beta}\right)}
$$

Proof: The LDG two-domain formulation is given in equation (40). Solving equation (40) for $\phi$ and $\psi$ yields the expressions

$$
\begin{aligned}
& \phi=\mathcal{D}_{l} \mathbf{U}+\frac{1}{\epsilon} \mathcal{P}_{l}^{-1}\left\{L_{10}\left[U_{i}-V_{i}\right] \quad \mathbf{e}_{\mathbf{i}_{-}}+L_{11}\left[(\phi)_{i}-(\psi)_{i}\right] \quad \mathbf{e}_{\mathbf{i}_{-}}\right\} \\
& \psi=\mathcal{D}_{r} \mathbf{V}+\frac{1}{\epsilon} \mathcal{P}_{r}^{-1}\left\{R_{10}\left[V_{i}-U_{i}\right] \quad \mathbf{e}_{\mathbf{i}_{+}}+R_{11}\left[(\psi)_{i}-(\phi)_{i}\right] \quad \mathbf{e}_{\mathbf{i}_{+}}\right\}
\end{aligned}
$$

while, solving equation (48) for the interface values yields the expressions

$$
\begin{aligned}
& \phi_{i}=\mathbf{e}_{\mathbf{i}_{-}}{ }^{T} \phi=\left.\left(\mathcal{D}_{l} \mathbf{U}\right)\right|_{i}+\frac{1}{\epsilon \alpha}\left\{L_{10}\left[U_{i}-V_{i}\right]+L_{11}\left[(\phi)_{i}-(\psi)_{i}\right]\right\} \\
& \psi_{i}=\mathbf{e}_{\mathbf{i}_{+}}{ }^{T} \psi=\left.\left(\mathcal{D}_{r} \mathbf{V}\right)\right|_{i}+\frac{1}{\epsilon \beta}\left\{R_{10}\left[V_{i}-U_{i}\right]+R_{11}\left[(\psi)_{i}-(\phi)_{i}\right]\right\}
\end{aligned}
$$

with the difference being

$$
\phi_{i}-\psi_{i}=\left.\left(\mathcal{D}_{l} \mathbf{U}\right)\right|_{i}-\left.\left(\mathcal{D}_{r} \mathbf{V}\right)\right|_{i}+\frac{1}{\epsilon}\left(\frac{L_{10}}{\alpha}+\frac{R_{10}}{\beta}\right)\left[U_{i}-V_{i}\right]+\frac{1}{\epsilon}\left(\frac{L_{11}}{\alpha}+\frac{R_{11}}{\beta}\right)\left[\phi_{i}-\psi_{i}\right]
$$


Rewriting equation (50) in terms of the difference $\phi_{i}-\psi_{i}$ yields

$$
\begin{gathered}
\phi_{i}-\psi_{i}=c_{1}\left[U_{i}-V_{i}\right] \quad+\quad c_{2}\left[\left.\left(\mathcal{D}_{l} \mathbf{U}\right)\right|_{i}-\left.\left(\mathcal{D}_{r} \mathbf{V}\right)\right|_{i}\right] \\
c_{1}=\frac{\frac{1}{\epsilon}\left(\frac{L_{10}}{\alpha}+\frac{R_{10}}{\beta}\right)}{1-\frac{1}{\epsilon}\left(\frac{L_{11}}{\alpha}+\frac{R_{11}}{\beta}\right)} \quad ; \quad c_{2}=\frac{1}{1-\frac{1}{\epsilon}\left(\frac{L_{11}}{\alpha}+\frac{R_{11}}{\beta}\right)}
\end{gathered}
$$

Substituting equations (48), (49) and (51) back into the original LDG formulation [equation (40)] yields the expression

$$
\begin{array}{rlr}
\mathcal{P}_{l} \mathbf{U}_{t}+a \mathcal{P}_{l} \mathcal{D}_{l} \mathbf{U} & =\epsilon \mathcal{P}_{l} \mathcal{D}_{l} \mathcal{D}_{l} \mathbf{U} \\
& +\left\{L_{00}\left[U_{i}-V_{i}\right]+L_{01}\left(c_{1}\left[U_{i}-V_{i}\right]+c_{2}\left[\left.\left(\mathcal{D}_{l} \mathbf{U}\right)\right|_{i}-\left.\left(\mathcal{D}_{r} \mathbf{V}\right)\right|_{i}\right]\right)\right\} & \mathcal{I}_{l} \mathbf{e}_{\mathbf{i}_{-}} \\
& +\left\{L_{10}\left[U_{i}-V_{i}\right]+L_{11}\left(c_{1}\left[U_{i}-V_{i}\right]+c_{2}\left[\left.\left(\mathcal{D}_{l} \mathbf{U}\right)\right|_{i}-\left.\left(\mathcal{D}_{r} \mathbf{V}\right)\right|_{i}\right]\right)\right\} & \mathcal{P}_{l} \mathcal{D}_{l} \mathcal{P}_{l}^{-1} \mathbf{e}_{\mathbf{i}_{-}} \\
\mathcal{P}_{r} \mathbf{V}_{t}+a \mathcal{P}_{r} \mathcal{D}_{r} \mathbf{V} & =\epsilon \mathcal{P}_{r} \mathcal{D}_{r} \mathcal{D}_{r} \mathbf{V} \\
& +\left\{R_{00}\left[V_{i}-U_{i}\right]+R_{01}\left(c_{1}\left[V_{i}-U_{i}\right]+c_{2}\left[\left.\left(\mathcal{D}_{r} \mathbf{V}\right)\right|_{i}-\left.\left(\mathcal{D}_{r} \mathbf{U}\right)\right|_{i}\right]\right)\right\} & \\
& +\left\{R_{10}\left[V_{i}-U_{i}\right]+R_{11}\left(c_{1}\left[V_{i}-U_{i}\right]+c_{2}\left[\left.\left(\mathcal{D}_{r} \mathbf{V}\right)\right|_{i}-\left.\left(\mathcal{D}_{r} \mathbf{U}\right)\right|_{i}\right]\right)\right\} & \mathcal{P}_{r} \mathcal{D}_{r} \mathcal{P}_{r}^{-1} \mathbf{e}_{\mathbf{i}_{+}} \\
&
\end{array}
$$

Simplifying equation (52) using the relations

$$
\begin{aligned}
\mathcal{P}_{l} \mathcal{D}_{l} \mathcal{P}_{l}^{-1} \mathbf{e}_{\mathbf{i}_{-}} & =-\mathcal{D}_{l}^{T} \mathbf{e}_{\mathbf{i}_{-}}+\frac{1}{\alpha} \mathbf{e}_{\mathbf{i}_{-}} \\
\mathcal{P}_{r} \mathcal{D}_{r} \mathcal{P}_{r}^{-1} \mathbf{e}_{\mathbf{i}_{+}} & =-\mathcal{D}_{r}^{T} \mathbf{e}_{\mathbf{i}_{-}}-\frac{1}{\beta} \mathbf{e}_{\mathbf{i}_{+}}
\end{aligned}
$$

yields

$$
\begin{aligned}
& \mathcal{P}_{l} \mathbf{U}_{t}+a \mathcal{P}_{l} \mathcal{D}_{l} \mathbf{U}-\epsilon \mathcal{P}_{l} \mathcal{D}_{l} \mathcal{D}_{l} \mathbf{U}=\left[\begin{array}{l}
L_{00}+L_{01} c_{1} \\
\frac{L_{10}}{\alpha}+\frac{L_{11} c_{1}}{\alpha}
\end{array}\right] \quad\left[\begin{array}{lll}
U_{i}-V_{i} & \mathcal{I}_{l} \mathbf{e}_{\mathbf{i}_{-}}
\end{array}\right. \\
& +\left[\begin{array}{llllll}
0 & + & L_{01} c_{2} \\
0 & + & \frac{L_{11} c_{2}}{\alpha}
\end{array}\right] \quad\left[\left.\left(\mathcal{D}_{l} \mathbf{U}\right)\right|_{i}-\left.\left(\mathcal{D}_{r} \mathbf{V}\right)\right|_{i}\right] \quad \mathcal{I}_{l} \mathbf{e}_{\mathbf{i}_{-}} \\
& +\left[\begin{array}{cccc}
0 & + & 0 \\
-L_{10} & - & L_{11} c_{1}
\end{array}\right] \quad\left[U_{i}-V_{i}\right] \quad \mathcal{D}_{l}^{T} \mathbf{e}_{\mathbf{i}_{-}} \\
& +\left[\begin{array}{cccc}
0 & + & 0 \\
0 & - & L_{11} c_{2}
\end{array}\right]\left[\left.\left(\mathcal{D}_{l} \mathbf{U}\right)\right|_{i}-\left.\left(\mathcal{D}_{r} \mathbf{V}\right)\right|_{i}\right] \quad \mathcal{D}_{l}^{T} \mathbf{e}_{\mathbf{i}_{-}}
\end{aligned}
$$

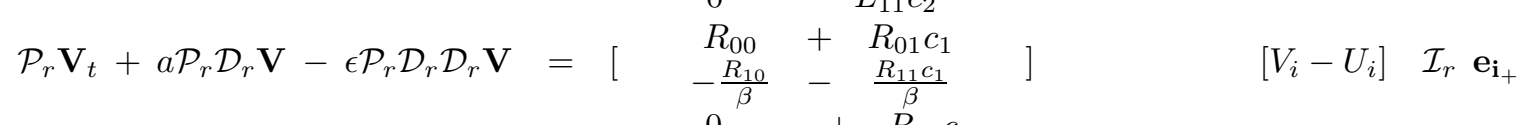

$$
\begin{aligned}
& +\left[\begin{array}{lllllll}
0 & + & R_{01} c_{2} \\
0 & - & \frac{R_{11} c_{2}}{\beta}
\end{array}\right]\left[\left.\left(\mathcal{D}_{r} \mathbf{V}\right)\right|_{i}-\left.\left(\mathcal{D}_{r} \mathbf{U}\right)\right|_{i}\right] \quad \mathcal{I}_{r} \mathbf{e}_{\mathbf{i}_{+}} \\
& +\left[\begin{array}{cccc}
0 & + & 0 \\
-R_{10} & - & R_{11} c_{1}
\end{array}\right] \quad\left[V_{i}-U_{i}\right] \quad \mathcal{D}_{r}^{T} \mathbf{e}_{\mathbf{i}_{+}} \\
& +\left[\begin{array}{ccc}
0 & + & 0 \\
0 & - & R_{11} c_{2}
\end{array}\right]\left[\left.\left(\mathcal{D}_{r} \mathbf{V}\right)\right|_{i}-\left.\left(\mathcal{D}_{r} \mathbf{U}\right)\right|_{i}\right] \quad \mathcal{D}_{r}^{T} \mathbf{e}_{\mathbf{i}_{+}}
\end{aligned}
$$

Comparing equation (53) with the original primal scheme given in equation (12) gives the desired result.

Remark. Note that the primal coefficients are not constants, but rather change with grid resolution. For example, the new parameter

$$
l_{00}=L_{00}+L_{01} c_{1}+\frac{L_{10}}{\alpha}+\frac{L_{11} c_{1}}{\alpha}
$$

given in equation (46) is inversely proportional to the grid spacing $\Delta x$ since $\alpha \propto \Delta x$, and becomes larger as the grid is refined.

It is reasonable to ask whether the stability constraints (29) for the primal method are preserved for methods satisfying the LDG stability constraints (42) and (43).

Theorem 7 Stability (in the $\mathcal{P}$-norm) in the LDG variables is a sufficient condition for stability (in the $\mathcal{P}$-norm) in the primal variables.

Proof : We begin by assuming that the LDG variables $L_{00} \rightarrow R_{11}$ are chosen such that the conservation conditions

$$
L_{00}=R_{00}+a, \quad L_{01}=R_{01}-\epsilon
$$


are satisfied, and that values of the parameters $S_{1}$ and $S_{2}$ satisfy the constraint equations

$$
0 \leq\left[S_{1}+S_{2}\right] \quad ; \quad \frac{\left[S_{1}-S_{2}\right]^{2}}{2\left[S_{1}+S_{2}\right]} \leq 1
$$

and

$$
L_{00} \leq \frac{a}{2}-\frac{\left[T_{1}+T_{2}\right]^{2}}{8[(\alpha+\beta) \epsilon]\left[S_{1}+S_{2}\right]}-\frac{\left[T_{1}-T_{2}+\frac{\left[S_{1}-S_{2}\right]\left[T_{1}+T_{2}\right]}{2\left(S_{1}+S_{2}\right)}\right]^{2}}{4(\alpha+\beta) \epsilon\left[1-\frac{\left[S_{1}-S_{2}\right]^{2}}{2\left[S_{1}+S_{2}\right]}\right]}
$$

Substitution of the transformation relations given by equation (46) into the primal conservations conditions $l_{00}=$ $r_{00}+a$; and $l_{01}=r_{01}-\epsilon$, yields the conservation equations based on the LDG variables;

$$
\begin{aligned}
l_{00}-r_{00}-a & = & 0 & \rightarrow & \left(L_{00}-R_{00}-a\right)+\frac{4\left(\alpha R_{10}+\beta L_{10}\right)\left(L_{01}-R_{01}+\epsilon\right)}{\epsilon\left[(\alpha-\beta)^{2}+4(\alpha+\beta)\left(\alpha S_{1}+\beta S_{2}\right)\right]} & =0 \\
l_{01}-r_{01}+\epsilon & = & 0 & \rightarrow & \frac{4(\alpha \beta)\left(L_{01}-R_{01}+\epsilon\right)}{\left[(\alpha-\beta)^{2}+4(\alpha+\beta)\left(\alpha S_{1}+\beta S_{2}\right)\right]} & =0
\end{aligned}
$$

while substitution of the transformation relations given by equation (46) into the primal and the stability constraints given by (29) yields the stability equations based on the LDG variables;

$$
\begin{aligned}
0 & \leq\left[s_{1}+s_{2}\right] \quad \rightarrow \quad 0 \leq \frac{2\left[\alpha^{2}\left(1+4 S_{1}\right)-\beta^{2}\left(1+4 S_{2}\right)\right]^{2}+64 \alpha^{2} \beta^{2}\left[S_{1}+S_{2}\right]\left[1-\frac{\left(S_{1}-S_{2}\right)^{2}}{2\left(S_{1}+S_{2}\right)}\right]}{\left[2\left((\alpha-\beta)^{2}+4(\alpha+\beta)\left(\alpha S_{1}+\beta S_{2}\right)\right)\right]^{2}} \\
0 & \leq\left[1-\frac{\left(s_{1}-s_{2}\right)^{2}}{2\left(s_{1}+s_{2}\right)}\right] \rightarrow \quad \rightarrow \quad 0 \leq \frac{32 \alpha^{2} \beta^{2}\left[S_{1}+S_{2}\right]\left[1-\frac{\left(S_{1}-S_{2}\right)^{2}}{2\left(S_{1}+S_{2}\right)}\right]}{\left[\alpha^{2}\left(1+4 S_{1}\right)-\beta^{2}\left(1+4 S_{2}\right)\right]^{2}}
\end{aligned}
$$

and

$$
\begin{gathered}
l_{00} \leq \frac{a}{2}-\frac{\left[t_{1}+t_{2}\right]^{2}}{8[(\alpha+\beta) \epsilon]\left[s_{1}+s_{2}\right]}-\frac{\left[t_{1}-t_{2}+\frac{\left[s_{1}-s_{2}\right]\left[t_{1}+t_{2}\right]}{2\left(s_{1}+s_{2}\right)}\right]^{2}}{4(\alpha+\beta) \epsilon\left[1-\frac{\left[s_{1}-s_{2}\right]^{2}}{2\left[s_{1}+s_{2}\right]}\right]} \\
\rightarrow \\
L_{00} \leq \frac{a}{2}-\frac{\left[T_{1}+T_{2}\right]^{2}}{8[(\alpha+\beta) \epsilon]\left[S_{1}+S_{2}\right]}-\frac{\left[T_{1}-T_{2}+\frac{\left[S_{1}-S_{2}\right]\left[T_{1}+T_{2}\right]}{2\left(S_{1}+S_{2}\right)}\right]^{2}}{4(\alpha+\beta) \epsilon\left[1-\frac{\left[S_{1}-S_{2}\right]^{2}}{2\left[S_{1}+S_{2}\right]}\right.}
\end{gathered}
$$

Thus, if the original scheme written in the LDG variables satisfies the required conservation and stability constraints, then it also satisfies the corresponding conservation and stability constraints written in the primal variables.

Remark: It has not been established whether stability (in the $\mathcal{P}$-norm) in the LDG variables is a necessary condition for stability (in the $\mathcal{P}$-norm) in the primal variables.

\section{Formal Accuracy}

We derive error estimates for the semi-discrete discretization method given by equation (12), under the assumption that $p \leq 1$. Extensive analysis of the accuracy of FEM is available in the literature (see for example the work of Arnold et al. [3]). Thus, the focus of attention herein is high-order finite difference methods.

Define the vectors $\mathbf{u}=\left[u\left(x_{0}, t\right), \cdots, u\left(x_{n}, t\right)\right]^{T}, \mathbf{u}_{\mathbf{x}}=\left[u_{x}\left(x_{0}, t\right), \cdots, u_{x}\left(x_{n}, t\right)\right]^{T}$, and $\mathbf{u}_{\mathbf{x x}}=\left[u_{x x}\left(x_{0}, t\right), \cdots, u_{x x}\left(x_{n}, t\right)\right]^{T}$, to be the projected values of the exact solution and derivatives, defined in the left domain at gridpoints $\mathbf{x}$. Similarly, define the vectors $\mathbf{v}, \mathbf{v}_{\mathbf{x}}$ and $\mathbf{v}_{\mathbf{x} \mathbf{x}}$ in the right domain. With these definitions, the continuous problem (9) can be represented in vector nomenclature as

$$
\begin{array}{rlrllll}
\mathbf{u}_{t}+a \mathbf{u}_{x}-\epsilon \mathbf{u}_{x x} & = & l_{00}\left[u_{i}-v_{i}\right] & \mathcal{P}_{l}^{-1} \mathcal{I}_{l} \mathbf{e}_{\mathbf{i}_{-}} & + & l_{01}\left[u_{x}-v_{x}\right]_{i} & \mathcal{P}_{l}^{-1} \mathcal{I}_{l} \mathbf{e}_{\mathbf{i}_{-}} \\
& + & l_{10}\left[u_{i}-v_{i}\right] & \mathcal{P}_{l}^{-1} \mathcal{D}_{l}^{T} \mathbf{e}_{\mathbf{i}_{-}} & + & l_{11}\left[u_{x}-v_{x}\right]_{i} & \mathcal{P}_{l}^{-1} \mathcal{D}_{l}^{T} \mathbf{e}_{\mathbf{i}_{-}} \\
\mathbf{u}(x, 0) & = & \mathbf{0 .} & & & & \\
& & & & & & \\
\mathbf{v}_{t}+a \mathbf{v}_{x}-\epsilon \mathbf{v}_{x x} & = & r_{00}\left[v_{i}-u_{i}\right] & \mathcal{P}_{r}^{-1} \mathcal{I}_{r} \mathbf{e}_{\mathbf{i}_{+}} & + & r_{01}\left[v_{x}-u_{x}\right]_{i} & \mathcal{P}_{r}^{-1} \mathcal{I}_{r} \mathbf{e}_{\mathbf{i}_{+}} \\
& + & r_{10}\left[v_{i}-u_{i}\right] & \mathcal{P}_{r}^{-1} \mathcal{D}_{r}^{T} \mathbf{e}_{\mathbf{i}_{+}} & + & r_{11}\left[v_{x}-u_{x}\right]_{i} & \mathcal{P}_{r}^{-1} \mathcal{D}_{r}^{T} \mathbf{e}_{\mathbf{i}_{+}} \\
\mathbf{v}(x, 0) & = & \mathbf{0 .} & & & &
\end{array}
$$


with zero initial data, neglected outer boundary conditions and source term $F(\mathbf{x}, t)$. Note that the interface terms $\left[u_{i}-v_{i}\right]$ and $\left[u_{x}-v_{x}\right]_{i}$ are identically zero for the exact solution, but have been added to the right hand side of equation (57) to facilitate subsequent manipulations.

Substituting the discrete accuracy relations $\mathbf{u}_{x}=\mathcal{D}_{l} \mathbf{u}+\mathbf{T}_{\mathbf{e} 1 l}$ and $\mathbf{u}_{x x}=\mathcal{D}_{l} \mathcal{D}_{l} \mathbf{u}+\mathbf{T}_{\mathbf{e} 2 l}$ [see definitions (4) and (7)], into equation (57) yields

$$
\begin{aligned}
& \mathbf{u}_{t}+a \mathcal{D}_{l} \mathbf{u}-\epsilon \mathcal{D}_{l} \mathcal{D}_{l} \mathbf{u}=l_{00}\left[u_{i}-v_{i}\right] \quad \mathcal{P}_{l}^{-1} \mathcal{I}_{l} \mathbf{e}_{\mathbf{i}_{-}}+l_{01}\left[\left(\mathcal{D}_{l} \mathbf{u}\right)_{i}-\left(\mathcal{D}_{r} \mathbf{v}\right)_{i}\right] \quad \mathcal{P}_{l}^{-1} \mathcal{I}_{l} \mathbf{e}_{\mathbf{i}_{-}} \\
& +l_{10}\left[u_{i}-v_{i}\right] \mathcal{P}_{l}^{-1} \mathcal{D}_{l}^{T} \mathbf{e}_{\mathbf{i}_{-}}+l_{11}\left[\left(\mathcal{D}_{r} \mathbf{v}\right)_{i}-\left(\mathcal{D}_{l} \mathbf{u}\right)_{i}\right] \quad \mathcal{P}_{l}^{-1} \mathcal{D}_{l}^{T} \mathbf{e}_{\mathbf{i}_{-}}
\end{aligned}
$$

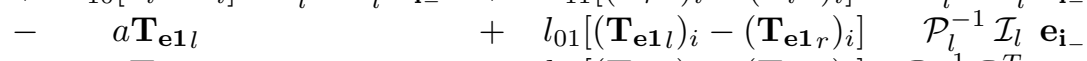

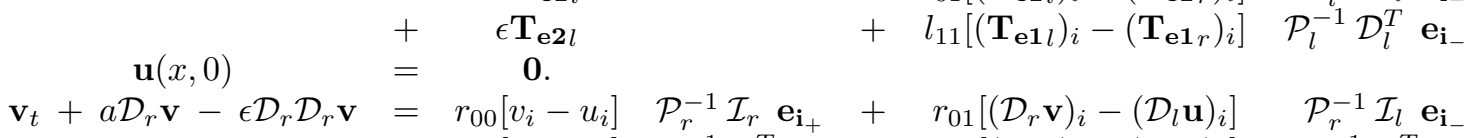

$$
\begin{aligned}
& +r_{10}\left[v_{i}-u_{i}\right] \mathcal{P}_{r}^{-1} \mathcal{D}_{r}^{T} \mathbf{e}_{\mathbf{i}_{+}}+r_{11}\left[\left(\mathcal{D}_{l} \mathbf{u}\right)_{i}-\left(\mathcal{D}_{r} \mathbf{v}\right)_{i}\right] \quad \mathcal{P}_{r}^{-1} \mathcal{D}_{l}^{T} \mathbf{e}_{\mathbf{i}_{-}}
\end{aligned}
$$

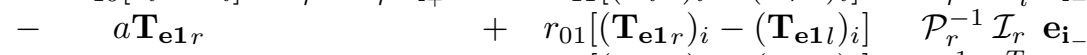

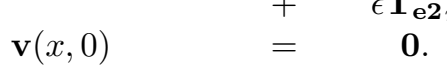

$$
\begin{aligned}
& +r_{11}\left[\left(\mathbf{T}_{\mathbf{e} 1 r}\right)_{i}-\left(\mathbf{T}_{\mathbf{e} 1 l}\right)_{i}\right] \quad \mathcal{P}_{r}^{-1} \mathcal{D}_{r}^{T} \mathbf{e}_{\mathbf{i}_{-}}
\end{aligned}
$$

Next, define the semi-discrete error vectors $\boldsymbol{\Xi}_{\mathbf{l}}=\mathbf{U}-\mathbf{u}$ and $\boldsymbol{\Xi}_{\mathbf{r}}=\mathbf{V}-\mathbf{v}$ in the left and right domains, respectively. Pre-multiplying by $\mathcal{P}^{-1}$ and subtracting equations (57) and (58) produces an evolution equation for the error vectors. Specifically, the collocation error of equation (9) written in primal form is

$$
\begin{aligned}
& \frac{\partial \boldsymbol{\Xi}_{1}}{\partial t}+a \mathcal{D}_{l} \boldsymbol{\Xi}_{\mathbf{l}}-\epsilon \mathcal{D}_{l} \mathcal{D}_{l} \boldsymbol{\Xi}_{\mathbf{l}}=l_{00}\left[\Xi_{l i}-\Xi_{r i}\right] \quad \mathcal{P}_{l}^{-1} \mathcal{I}_{l} \mathbf{e}_{\mathbf{i}_{-}}+l_{01}\left[\left(\mathcal{D}_{l} \Xi_{l}\right)_{i}-\left(\mathcal{D}_{r} \Xi_{r}\right)_{i}\right] \quad \mathcal{P}_{l}^{-1} \mathcal{I}_{l} \mathbf{e}_{\mathbf{i}_{-}} \\
& +l_{10}\left[\Xi_{l i}-\Xi_{r i}\right] \quad \mathcal{P}_{l}^{-1} \mathcal{D}_{l}^{T} \mathbf{e}_{\mathbf{i}_{-}}+l_{11}\left[\left(\mathcal{D}_{l} \Xi_{l}\right)_{i}-\left(\mathcal{D}_{r} \Xi_{r}\right)_{i}\right] \quad \mathcal{P}_{l}^{-1} \mathcal{D}_{l}^{T} \mathbf{e}_{\mathbf{i}_{-}} \\
& +\quad a \mathbf{T}_{\mathbf{e} 1 l} \quad-l_{01}\left[\left(\mathbf{T}_{\mathbf{e} 1 l}\right)_{i}-\left(\mathbf{T}_{\mathbf{e} 1 r}\right)_{i}\right] \quad \mathcal{P}_{l}^{-1} \mathcal{I}_{l} \mathbf{e}_{\mathbf{i}_{-}}
\end{aligned}
$$

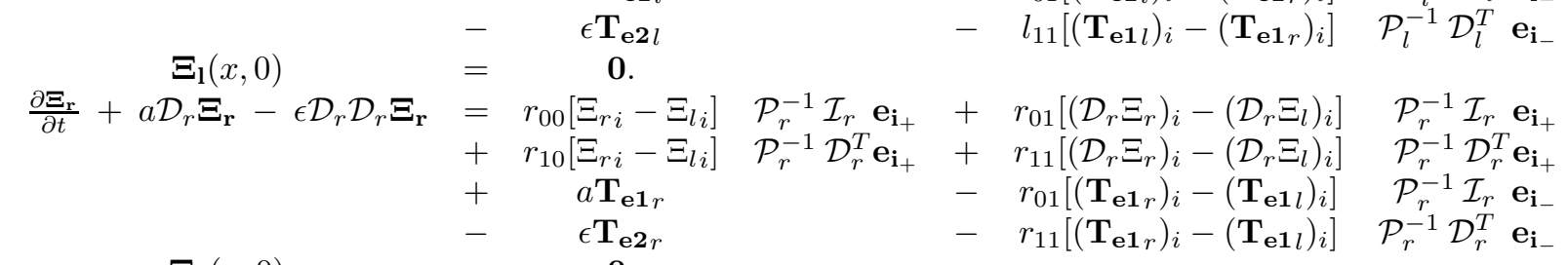

Four distinct types of discretization errors appear in equation (59). The first two truncation error vectors: $\mathbf{T}_{\mathbf{e} 1}$ and $\mathbf{T}_{\mathbf{e} 2}$, arise from errors associated with the approximation of the first and second derivative terms, respectively. Like conventional HOFDM operators, they are seldom uniformly accurate throughout the domain. Points near boundaries are commonly discretized less accurately than those in the interior. Furthermore, the boundary stencils used in first and second difference operators are frequently of different orders.

The last two error vectors result from the interface penalty terms and have the forms: $\left[\left(\mathbf{T}_{\mathbf{e} 1}\right)_{i}-\left(\mathbf{T}_{\mathbf{e} \mathbf{1}}\right)_{i}\right] \mathcal{P}^{-1} \mathcal{I} \mathbf{e}_{\mathbf{i}}$ and $\left[\left(\mathbf{T}_{\mathbf{e} 1}\right)_{i}-\left(\mathbf{T}_{\mathbf{e} 1}\right)_{i}\right] \mathcal{P}^{-1} \mathcal{D}^{T} \mathbf{e}_{\mathbf{i}}$. Note that the accuracy of these vectors is influenced both by the truncation error of the interface derivative operators, $\left(\mathbf{T}_{\mathbf{e} 1 r}\right)_{i}$, and by the size of the penalty scaling terms. To assess the size of the scaling terms, first note that the differentiation operator and its transpose, $\left[\mathcal{D}\right.$ and $\mathcal{D}^{T}$ ] scale as $\mathcal{O}(\Delta x)^{-1}$. Further, since $\mathcal{Q}$ is unitless, and $\mathcal{D}=\mathcal{P}^{-1} \mathcal{Q}$, the inverse norm operator $\mathcal{P}^{-1}$ scales as $\mathcal{O}(\Delta x)^{-1}$. Thus, the last two error vectors scale as $\mathcal{O}(\Delta x)^{r-1}$ and $\mathcal{O}(\Delta x)^{r-2}$, respectively, where $r$ is the local accuracy of the interface derivative operator.

In summary, if interface penalties satisfy $p \leq 1$ then the error vectors in both domains have leading order truncation terms that scale as

$$
\begin{aligned}
& \mathbf{T}_{\mathbf{e} 1}=\left[\mathcal{O}\left(\Delta x^{r 1}\right), \cdots, \mathcal{O}\left(\Delta x^{r 1}\right) \quad, \mathcal{O}\left(\Delta x^{2 p}\right), \cdots, \mathcal{O}\left(\Delta x^{2 p}\right), \mathcal{O}\left(\Delta x^{r 1}\right) \quad, \cdots, \mathcal{O}\left(\Delta x^{r 1}\right)\right]^{T} \\
& \mathbf{T}_{\mathbf{e} 2}=\left[\mathcal{O}\left(\Delta x^{r 2}\right), \cdots, \mathcal{O}\left(\Delta x^{r 2}\right), \mathcal{O}\left(\Delta x^{2 p}\right), \cdots, \mathcal{O}\left(\Delta x^{2 p}\right), \mathcal{O}\left(\Delta x^{r 2}\right), \cdots, \mathcal{O}\left(\Delta x^{r 2}\right)\right]^{T} \\
& \left(\mathbf{T}_{\mathbf{e} 1}\right)_{i} \mathcal{P}^{-1} \mathcal{I}^{T} \mathbf{e}_{\mathbf{i}}=\left[\mathcal{O}\left(\Delta x^{r 1-1}\right), \cdots, \mathcal{O}\left(\Delta x^{r 1-1}\right), \quad 0 \quad, \cdots, \quad 0 \quad, \mathcal{O}\left(\Delta x^{r 1-1}\right), \cdots, \mathcal{O}\left(\Delta x^{r 1-1}\right)\right]^{T} \\
& \left(\mathbf{T}_{\mathbf{e} 1}\right)_{i} \mathcal{P}^{-1} \mathcal{D}^{T} \mathbf{e}_{\mathbf{i}}=\left[\mathcal{O}\left(\Delta x^{r 1-2}\right), \cdots, \mathcal{O}\left(\Delta x^{r 1-2}\right), \quad 0 \quad, \cdots, \quad 0 \quad, \mathcal{O}\left(\Delta x^{r 1-2}\right), \cdots, \mathcal{O}\left(\Delta x^{r 1-2}\right)\right]^{T}
\end{aligned}
$$

where the $r 1$ and $r 2$ exponents denote the boundary stencil order of accuracy in the first- and second-derivative operators, respectively. (Here, we assume the interior stencils to be the same order of accuracy for both the first- and second-derivative operators.) 
An error equation derived for the general penalty technique presented in equation (11) would involve considerably more error terms. For example, extending equation (59) to the case $p \leq 2$ would produce the following additional terms

$$
\begin{array}{lll}
l_{02} & \left(\mathbf{T}_{\mathbf{e} 2}\right)_{i} & \mathcal{P}^{-1} \mathcal{I}_{l}^{T} \mathbf{e}_{\mathbf{i}}=\mathcal{O}\left(\Delta x^{r 2-1}\right) \\
l_{12} & \left(\mathbf{T}_{\mathbf{e} 2}\right)_{i} & \mathcal{P}^{-1} \mathcal{D}_{l}^{1^{T}} \mathbf{e}_{\mathbf{i}}=\mathcal{O}\left(\Delta x^{r 2-2}\right) \\
l_{22} & \left(\mathbf{T}_{\mathbf{e} 2}\right)_{i} & \mathcal{P}^{-1} \mathcal{D}_{l}^{2} \mathbf{e}_{\mathbf{i}}=\mathcal{O}\left(\Delta x^{r 2-3}\right) \\
l_{21} & \left(\mathbf{T}_{\mathbf{e} 1}\right)_{i} & \mathcal{P}^{-1} \mathcal{D}_{l}^{2} \mathbf{e}_{\mathbf{i}}=\mathcal{O}\left(\Delta x^{r 1-3}\right) \\
l_{20} & & \mathcal{P}^{-1} \mathcal{D}_{l}^{2} \mathbf{e}_{\mathbf{i}}=\mathcal{O}\left(\Delta x^{2 p-3}\right)
\end{array}
$$

as well as similar expressions for $r_{02}, r_{12}, r_{22}, r_{21}, r_{20}$.

Local lower order error terms do not necessarily decrease the global formal accuracy. The impact of lower-order terms on global discretization accuracy is a long-standing area of research that dates back to the pioneering work of Gustafsson [14]. There it was established that hyperbolic operators could accommodate local terms one order lower than design order, while still maintaining formal accuracy. Recent work of Svärd and Nordström [34] has extended this result to include the impact of low-order stencils for parabolic problems. Their work on global accuracy is encapsulated in the following theorem.

Theorem 8 If (12) is a pointwise stable discretization of the continuous problem (9) for $\Delta x \leq \Delta x_{0}$, then with interior operators $\mathcal{D}$ and $\mathcal{D D}$ satisfying discretization orders of $2 p-2 \leq r 1, r 2 \leq 2 p$ at the boundary, and interface penalty terms satisfying discretization orders of $2 p-2 \leq r 1-2 \leq 2 p$, then the global order of accuracy of approximation (12) is $2 p$.

Proof : See Svärd and Nordström [34], Theorems (2.6) and (2.8), pp. 335-337 for the original theorems and proofs.

Now consider the discretization of equation (9) using a realistic combination of operators to determine the effects of each error term on global accuracy. Assume that the derivative operator $\mathcal{D}$ is defined as in equation (60) with $r 1=2 p-1$. Furthermore, assume that the second derivative operator is formed as the action of two first derivative operators $\mathcal{D D}$ so that $r 2=2 p-2$. Finally, assume that the derivative used in the penalty (obtained from the derivative operator $\mathcal{D}$ ) is $\mathcal{O}\left(\Delta x^{2 p-1}\right)$, and is used in all penalty terms where necessary. In this scenario, the derivative operators $\mathcal{D}$ and $\mathcal{D D}$ maintain the design accuracy $2 p$ of the method, as well as do the penalty terms producing errors of the form $\left(\mathbf{T}_{\mathbf{e} 1}\right)_{i} \mathcal{P}^{-1}$. The remaining penalty terms producing errors of the form $\mathcal{D}^{T} \mathbf{e}_{\mathbf{i}}$ decrease the formal accuracy to $2 p-1$, at least in principle.

Remark. Adding penalty terms of the form $\left(\mathbf{T}_{\mathbf{e} 1}\right)_{i} \mathcal{P}^{-1} \mathcal{D}^{T} \mathbf{e}_{\mathbf{i}}$ is ill advised on a theoretical basis, although experiments presented later show that these estimates are not always sharp. Furthermore, these experiments will show these terms to be computationally impractical.

Remark. Assume that $j^{\text {th }}$-derivative operator $\mathcal{D}^{j}, \quad 2 \leq j \leq p$ is $r_{j}$-order accurate at the interface, and has interface truncation error $\left.\mathbf{T}_{\mathbf{e}_{\mathbf{j}}}\right)_{i}$. Interface penalties using these derivative operators produce terms in the error equation of the form

$$
l_{j k}\left(\mathbf{T}_{\mathbf{e}_{\mathbf{j}}}\right)_{i} \mathcal{P}^{-1} \mathcal{D}^{k^{T}} \mathbf{e}_{\mathbf{i}}=\mathcal{O}\left(\Delta x^{r_{j}-(k+1)}\right)
$$

Remark. The reduction in accuracy is more severe for a hyperbolic equation [e.g. $\epsilon=0$ in equation (9)]. Specifically, the boundary / penalty discretization orders required to achieve formal accuracy satisfies $2 p-1 \leq r 1, r 2 \leq 2 p$. Thus, penalties of the form $\left(\mathbf{T}_{\mathbf{e} 1}\right)_{i} \mathcal{P}^{-1} \mathbf{e}_{\mathbf{i}}$ and $\left(\mathbf{T}_{\mathbf{e} 1}\right)_{i} \mathcal{P}^{-1} \mathcal{D}^{T} \mathbf{e}_{\mathbf{i}}$ decrease the formal accuracy to $2 p-1$ and $2 p-2$, respectively.

\section{Identification of schemes}

Specific values are assigned for the eighteen parameters used in the primal [equation (12)] and flux [equation (40)] formulations. Three popular schemes are identified, including two DGFEM and one strong form HOFDM. Each is shown to satisfy their respective stability constraints. All three scheme only penalize the solution and first derivative, so the parameters $l_{02}, l_{12}, l_{22}, l_{21}, l_{20}$ and $r_{02}, r_{12}, r_{22}, r_{21}, r_{20}$ are set to zero. 


\subsection{Carpenter, Nordström, Gottlieb [8]}

We begin with the scheme proposed by Carpenter et. al (CNG) [8] which is a special case of the primal formulation presented in equation (12). This scheme was originally derived in strong form in the context of HOFDM. This scheme [See reference [8], equation (7).] can be reproduced by assigning the following specific values to the penalty parameters;

$$
l_{10}=l_{11}=r_{10}=r_{11}=0 ; \quad l_{00}=\sigma_{1} ; \quad l_{01}=\epsilon \sigma_{2} ; \quad r_{00}=\sigma_{3} ; \quad r_{01}=\epsilon \sigma_{4} ;
$$

Substituting (62) into equation (29) yields the conditions

$$
\begin{gathered}
\sigma_{1}=\sigma_{3}+a \quad ; \quad\left[\sigma_{2}=\sigma_{4}-1\right] \\
0 \leq[(\alpha+\beta) \epsilon] \quad ; \quad[\epsilon(-\alpha+\beta)]^{2} \leq[(\alpha+\beta) \epsilon]^{2} \quad ; \quad \sigma_{1} \leq \frac{a}{2}-\epsilon\left[\frac{\sigma_{2}^{2}}{4 \beta}+\frac{\sigma_{4}^{2}}{4 \alpha}\right]
\end{gathered}
$$

Further, the stability conditions obtained in equation (63) and (64) are identical to those found in the original CNG work [e.g. equation (8) in reference [8]].

\subsection{Baumann, Oden [4]}

The scheme proposed by Baumann and Oden [4] is a special case of the primal formulation presented in equation (12). This scheme was originally developed in the weak form in the context of DGFEM. Shu presents in reference [30], an illustrative comparison between this scheme and the LDG scheme for the strictly parabolic case. Therein the Baumann-Oden scheme is presented in equations (5.1) and (5.2) and can be reproduced from equation (12) with the values

$$
l_{00}=r_{00}=l_{11}=r_{11}=0 \quad ; \quad l_{01}=-\frac{\epsilon}{2} ; \quad l_{10}=-\frac{\epsilon}{2} ; \quad r_{01}=+\frac{\epsilon}{2} ; \quad r_{10}=+\frac{\epsilon}{2}
$$

Taking the liberty to extend Shu's definition to include the possibility of convection terms, we define the BaumannOden scheme by assigning the following values to the coefficients in equation (12):

$$
l_{00}=r_{00}+a ; \quad l_{11}=r_{11}=0 ; \quad l_{01}=+\left(\beta-\frac{\epsilon}{2}\right) ; \quad l_{10}=-\left(\beta+\frac{\epsilon}{2}\right) ; \quad r_{01}=+\left(\beta+\frac{\epsilon}{2}\right) ; \quad r_{10}=-\left(\beta-\frac{\epsilon}{2}\right)
$$

Substituting (66) into equation (29) yields the conditions

$$
0 \leq[(\alpha+\beta) \epsilon] \quad ; \quad[\epsilon(-\alpha+\beta)]^{2} \leq[(\alpha+\beta) \epsilon]^{2} \quad ; \quad l_{00} \leq \frac{a}{2}
$$

\subsection{Local Discontinuous Galerkin [11]}

The scheme proposed by Cockburn and Shu [11] is special case of the flux formulation presented in equation (40). Once again, Shu presents in reference [30], the LDG scheme written strictly for the parabolic case. Therein the LDG scheme is presented in equations (4.1), (4.2), and (4.3) and can be reproduced from equation (40) with the values

$$
L_{00}=R_{00}=L_{11}=R_{11}=0 ; \quad L_{01}=-\frac{\epsilon}{2} ; \quad L_{10}=-\frac{\epsilon}{2} ; \quad R_{01}=+\frac{\epsilon}{2} ; \quad R_{10}=+\frac{\epsilon}{2}
$$

Again, taking the liberty to extend Shu's definition to include the possibility of convection terms, we define the collocation LDG scheme by assigning the following values to the coefficients in equation (40):

$$
L_{00}=R_{00}+a ; \quad L_{11}=R_{11}=0 ; \quad L_{01}=+\left(\beta-\frac{\epsilon}{2}\right) ; \quad L_{10}=-\left(\beta+\frac{\epsilon}{2}\right) ; \quad R_{01}=+\left(\beta+\frac{\epsilon}{2}\right) ; \quad R_{10}=-\left(\beta-\frac{\epsilon}{2}\right)
$$

Substituting (69) into equation (29) yields the conditions

$$
0 \leq[(\alpha+\beta) \epsilon] \quad ; \quad[\epsilon(-\alpha+\beta)]^{2} \leq[(\alpha+\beta) \epsilon]^{2} \quad ; \quad L_{00} \leq \frac{a}{2}
$$




\section{$5 \quad$ Numerical experiments}

\subsection{Test Problems}

\subsubsection{The One-Way Wave Equation}

The linear one-way wave equation is used to study the stability and accuracy of the new formulations in the absence of diffusion terms. The functional form is

$$
U_{t}+a U_{x}=0 \quad|x| \leq 1 \quad, 0 \leq t \leq 0.1
$$

with the exact solution

$$
U(x, t)=\cos [2 \pi(x-t)]
$$

The initial and boundary data coincide with the exact solution for all time.

The one-way wave equation is used to establish a baseline accuracy for each method, before moving on to the parabolic equation. This step is essential to establish the effects of the newly added terms that penalize the diffusive terms. Specifically, the new additional terms could destroy the accuracy of the advection term, not just diffusion. Comparing the two results allows us to determine the source of error.

This study of the first order one-way wave equation raises a subtle point regarding imposition of discrete interface conditions; specifically, that derivative fluxes can be penalized between elements, despite the fact that wellposedness requires only one interface condition. Thus, the diffusive interface penalty can be thought of as an alternative discrete stencil, one that weakly enforces first derivative continuity across the interface. An a priori stability estimate and accurate interface data guarantee the validity of these penalties.

\subsubsection{Linear Burgers' Equations}

The linear Burgers' equation tests a combination of the advection and diffusion terms. Five distinct values of the diffusion parameter $\epsilon$ are used to deduce the effects of the penalty as the problem becomes diffusion dominated. The functional form is

$$
U_{t}+a U_{x}=\epsilon U_{x x} \quad|x| \leq 1 \quad, 0 \leq t \leq 0.1
$$

with the exact solution

$$
U(x, t)=\exp \left[-\epsilon b^{2} t\right] \cos [-2 \pi(x-t)]
$$

with initial and boundary data that coincides with that of the exact solution. The equation parameters used are $a=2$ and $b=2 \pi$ in all cases. Five values of the parameter $\epsilon$ are used: $\epsilon=1, \frac{2}{5}, 0.1 \frac{2}{50}$, and 0.01 .

\section{$5.2 \quad$ Test Schemes}

Two finite difference and four spectral operators are tested using the linear wave and linear Burgers' equation. The finite difference operators are the $4^{\text {th }}$ and $6^{\text {th }}$ order central difference operators using SBP closures at the boundaries. All finite difference simulations utilize a uniform grid within each element, although the element size is allowed to vary. Block-norm boundary closures are used that are $3^{r d}$ - and $5^{\text {th }}$-order accurate [6], respectively. The boundary closure formulas are those reported in reference [6]. The spectral operators are conventional collocation operators defined using the Gauss-Lobatto-Chebyshev nodal points. Polynomial orders in the range $2 \leq p \leq 5$ are used (corresponding to elements having three to six nodal points). The penalties are implemented such that the scheme is equivalent to a Legendre collocation scheme (see Carpenter and Gottlieb [7]). The time advancement scheme used in all cases is the five-stage fourth-order Runge-Kutta (RK) scheme [5]. The timestep is chosen such that the temporal error estimate is independent of further timestep reduction. The magnitude of the temporal error is approximately $10^{-12}$, and is obtained by comparing the fourth-order solution with an embedded third-order solution. Thus, the spatial error is the dominant component of error in the simulations. 


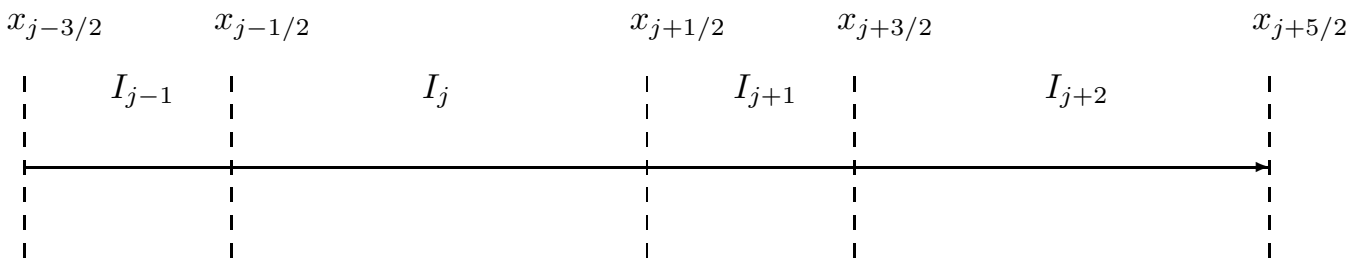

Figure 2: Schematic depicting two periods of the recursive element pattern used in the nonuniform grid accuracy studies.

\subsection{Test Matrix}

An extensive test matrix is used to distinguish different properties of the new formulations. The matrix includes 1) four geometry definitions, and 2) multiple values of three distinct interface penalty parameters. The geometric definitions included all permutations of uniform and nonuniform element distributions on both periodic and nonperiodic intervals, specifically; 1) uniform-periodic, 2) nonuniform-periodic, 3) uniform-nonperiodic, and 4) nonuniform-nonperiodic. The nonuniform grids are generated using elements that alternate in size with a ratio of $2: 1$. Figure (2) shows a schematic depicting two periods of the $2: 1$ nonuniform grid used in the study.

An a priori study is performed, comparing the accuracy of the methods on each of the four different geometries. The results for these studies are available in reference [9]. The most challenging test cases (hyperbolic or parabolic) are those that were run on the nonuniform grid, with nonperiodic boundary conditions. Thus, the nonuniform grid with nonperiodic boundary conditions is used almost exclusively in this work.

The interface penalties are parametrized using three independent variables as (here written for the primal formulation),

$$
\begin{gathered}
l_{00}=\frac{a}{2}(1-\alpha) ; \quad r_{00}=\frac{a}{2}(-1-\alpha) ; \quad \alpha \geq 0 \\
l_{01}=+\left(\beta-\frac{\epsilon}{2}\right) ; \quad l_{10}=-\left(\beta+\frac{\epsilon}{2}\right) ; \quad r_{01}=+\left(\beta+\frac{\epsilon}{2}\right) ; \quad r_{10}=-\left(\beta-\frac{\epsilon}{2}\right) \\
l_{11}=r_{11}=\gamma ; \quad \gamma \geq 0
\end{gathered}
$$

The parameter $\alpha$ adjusts the contribution of the Dirichlet penalty between the left and right states. The special value $\alpha=0$ admits no dissipation at the interface, thus producing a skew-symmetric matrix in the $\mathcal{P}$-norm. The value $\alpha=1$ produces a fully upwind flux. Two values of the parameter $\alpha$ are tested: $\alpha=0$ and $\alpha=1$.

The parameters $\beta$ and $\gamma$ adjust the penalty on the derivative fluxes. For $\beta$ the relationship between the values of $l_{01}, l_{10}, r_{01}$, and $r_{10}$, greatly simplifies the energy estimate by producing the conditions $t_{1}=t_{2}=0$ in the estimate. This combination of parameters adds no dissipation to the energy estimate. Note that the value of $\beta$ can be chosen independent from $\epsilon$, and can be nonzero for values of $\epsilon=0$. Two values of the parameter $\beta$ are tested: $\beta=0$ and $\beta=\frac{1}{4}$. The parameter $\gamma$ adjusts the contribution from the derivative fluxes and produces contributions to the energy estimate that are strictly dissipative. Four values of $\gamma$ are tested: $\gamma=0, \gamma=10^{-4}, \gamma=10^{-3}$ and $\gamma=10^{-2}$.

All studies include a comparison of the convergence rate of each scheme. A grid refinement exercise involving approximately 40 grid densities is performed for each set of parameters. The convergence rate is determined by fitting a "least-squares" curve through the error data.

\subsection{Results}

\subsubsection{Finite Difference: One-Way Wave and Linear Burgers' Equation}

Figure (3) compares the convergence behavior of the $4^{\text {th }}$ - and $6^{\text {th }}$-order finite difference schemes. The model problem used is the linear one-way wave equation. Shown are the results obtained on a nonuniform grid with nonperiodic boundary conditions. Seven sets of interface parameters are compared, and in all cases, design order convergence is 
observed $\left(4^{\text {th }}\right.$ - and $6^{\text {th }}$-order, respectively). Note that imposing the penalty on the derivative-transpose terms $\left(l_{11}\right.$, and $r_{11}$ ), in contrast to theoretical predictions, does not decrease the formal accuracy of the schemes.

Figure (4) compares the convergence behavior of the $4^{t h}$ and $6^{t h}$-order schemes on the linear Burgers' equation. Both the flux (LDG) and the primal (B-O) forms of the penalty are presented in this study. Note that the parameter $\beta$ is not scaled with the parameter $\epsilon$. In all cases, design order is achieved, implying that increased levels of interface coupling has little impact on formal solution accuracy. Two other values of the physical viscosity $\epsilon$ were also simulated (but not shown), and similar trends were observed in both cases.

An anomalous behavior is observed in the convergence of the $4^{\text {th }}$-order simulations. While the slope remains close to 4 , a deflection in convergence behavior is observed at an error of approximately $10^{-8}$. The exact cause of this behavior is not known.

All simulations are performed with an explicit RK scheme, and the efficiency of the runs is strongly influenced by spurious eigenvalues generated by the interface coupling terms. For the parameters tested, little sensitivity is observed for the $\alpha$ parameter. A significant decrease in the max-CFL is observed when large values of the $\beta$ parameter are used. Even small values of the $\gamma$ parameter render the explicit method almost useless, a consequence of "interface" eigenvalues that progressively extend further towards negative infinity as $\gamma$ is increased. An implicit method would certainly be needed if large values of $\gamma$ are used at the interfaces. The max-CFL never increases beyond the baseline value when using $\alpha, \beta$ or $\gamma$.

Finally, both uniform and nonuniform grids are used in this study, as are periodic and nonperiodic geometries. Neither permutation changed in any way the general convergence behavior of the schemes presented in figures (3)-(4).

\subsubsection{Spectral Collocation: One-Way Wave and Linear Burgers' Equation}

For the one-way wave equation we only use the primal (BO) formulation. The same $\alpha, \beta, \gamma$ parameter studies performed for the finite difference cases are repeated using the spectral collocation methods. Polynomial orders ranging from $p=2$ to $p=5$ are compared on a nonuniform grid with nonperiodic boundaries. The convergence rate as determined by a least-squares fit of error data, is reported in the tabular comparisons. Three different measures of error are used in the tabular comparisons: 1) the $L_{2}$-norm of the solution error, 2) the $L_{\infty}$-norm, and 3) the integral norm, formed using the integration matrix $\mathcal{P}$.

Table (1), shows the convergence results for four permutations of $\alpha$ and $\beta$, and polynomial orders $p=2,3,4,5$, at values of $\gamma$ in the range $[0.0 \leq \gamma \leq 0.025]$. Three distinct convergence rates emerge in this study. We begin by comparing the cases run with $\gamma=0$. The case $\alpha=1, \beta=0$ converges at the optimal rate of $p+1$ for all polynomial orders. Changing the value to $\beta=\frac{1}{4}$ reduces the formal convergence rate to $p$. Next, imposing a nonzero value of the parameter $\gamma$ decreases the convergence rate of the optimal case $(\alpha=1, \beta=0)$ to what appears to be $p+\frac{1}{2}$. Strangely enough, imposing nonzero values for $\gamma$ increases the accuracy of the suboptimal cases for which $\beta=\frac{1}{4}$.

Once again, all these studies were run with an explicit RK scheme that is subject to a finite maximum stability condition. The interface penalties have a profound influence on the placement of the maximum eigenvalues, and therefore the maximum allowable timestep $\left[(\Delta t)_{\max }\right]$. The parameter $\beta$ decreased the $(\Delta t)_{\max }$ significantly for $\mathcal{O}(1)$ values. The parameter $\gamma$ rendered the explicit time advancement scheme almost useless, by reducing the $(\Delta t)_{\max }$ by several orders of magnitude.

Two studies are performed on the linear Burgers' equation: one using the primal formulation (e.g. BaumannOden), and one using the flux formulation (e.g. LDG). We begin with a study comparing the influence of the equation parameter $\epsilon$ on the convergence rate of the Baumann-Oden scheme.

Table (2) compares the convergence of the B-O scheme for values of the physical parameter $\epsilon=1.00,0.10,0.01$. (The $L_{2}$-norm of the derivative error is included in the results along with the $L_{2}$-norm, the $L_{\infty}$-norm, and the $\mathcal{P}$ integral norm.) The cases with diffusive parameter $\epsilon=1.0$, are dominated by the parabolic nature of the equations, while as $\epsilon$ becomes smaller, the character of the equation becomes increasingly "hyperbolic".

Suboptimal convergence is generally observed for all polynomial orders and schemes. The exceptional cases are when the Dirichlet boundary and interface terms are fully upwinded $(\alpha=1)$ and the Neumann derivative terms are averaged $(\beta=0)$. For this set of parameters, polynomials of odd order $(p=3,5)$ converge at an optimal rate of $p+1$ for the solution and $p$ for the derivative. This result is consistent with that shown by Shu [30] for the strictly parabolic case using DGFEM. As $\epsilon$ decreases from the baseline $\epsilon=1$, the convergence rate increases (unexpectedly), 
for all schemes and all polynomial orders with the exception of those that are already optimal.

The convergence behavior of the B-O formulation is unpredictable. In contrast, when the linear Burgers' equation is simulated with the LDG scheme, optimal convergence is achieved for every combination of physical/numerical parameters. Table (3) shows the convergence behavior of the LDG scheme for the same four parameter settings, and four polynomial orders. The LDG scheme converges for the solution at a rate of $p+1$, while the derivative converges at a rate $p$ for all polynomial orders $p$. Little influence is observed when the values of the parameters $\alpha$ or $\beta$ are adjusted. These results for the LDG scheme are in slight disagreement with those presented by Shu [30] for the DGFEM and a strictly parabolic equation. There it is shown that optimal convergence is only achieved for the specific values of the parameter $\beta= \pm \frac{1}{2}$.

The formal accuracy of the LDG scheme is clearly superior to that of the B-O formulation. Figures (5), and (6) compare the absolute accuracy of both formulations, all permutations of $\alpha$ and $\beta$, and the physical viscosity $\epsilon=1$. The LDG formulation significantly outperforms the B-O scheme for even order polynomials, but is less advantageous for odd order polynomials (for which the B-O scheme is optimally convergent). Both the B-O and LDG classes of schemes, exhibit little sensitivity to the parameters $\alpha=0,1$ and $\beta=0 / 4,1 / 4$. This result is surprising for the B-O schemes, considering that some are suboptimal for various parameter combinations. Note, however, that absolute accuracy depends on both the order of and size of the truncation terms. Many of the lower-order combinations have smaller truncation terms, which results in better accuracy at coarse tolerances. Note that figure (6) also shows the $4^{\text {th }}$-order finite difference result, which is more accurate than either the B-O or LDG formulation.

Figure (7) combines all the polynomial orders in to one plot. For clarity, only the cases with interface parameters $\alpha=1$ and $\beta=0$, are presented. Recall that this parameter combination results in an upwind Dirichlet flux and a centered Neumann flux at the interfaces, and resulted in optimal convergence for odd-orders with the B-O scheme. The LDG scheme is significantly more accurate than the B-O scheme for even order polynomials, while they are nearly indistinguishable for odd order polynomials. Note that the convergence behavior of the LDG formulation is dual valued for even order polynomials. Specifically, the simulations with an even number of elements superconverges initially, while those with an odd number of elements converge as expected.

\section{Discussion}

The present work generalizes the penalty formulation proposed by the present authors (see reference [8]) to couple adjoining SBP operators. The resulting formulation provides a "plethora" of adjustable parameters that generate schemes with remarkably diverse numerical properties. Broad generalities regarding the various formulations include: 1 ) the accuracy of the finite difference methods is neither sensitive to the interface parameters $\alpha, \beta, \gamma$, nor sensitive to the formulation type (e.g. primal or flux), 2) the accuracy of the spectral collocation methods is weakly sensitive to the interface parameters, 3 ) the accuracy of the LDG flux formulation greatly exceeds that of the BO primal formulation for spectral collocation, 4) second derivative penalty terms greatly increase the spectral radius of the semi-discrete operator.

While the model problems studied herein provide little evidence of the virtues of higher derivative interface coupling, it seems premature to disregard them from further consideration before performing more thorough testing. One needed test (suggested by a reviewer) is to quantify the impact of second derivative penalties on the smoothness of all derivatives across the interface, and to establish whether increased levels of smoothness is beneficial. Another needed test is to quantify the impact of high derivative penalties on the spectral radius of the discrete operator. Indeed, increases in the spectral radius adversely impact both explicit and implicit time advancement techniques.

The present derivations and analysis, focus entirely on the 1D, scalar, constant coefficient, advection-diffusion equation, although generalization to 3D is conceptually "trivial" based on tensor product formulations and algebra [26], albeit difficult in practical terms. (Not to make light of the significant impediments encountered when building 3D software: grid generation, solution and geometry singularities, nonlinear stability, etc.) Generalization to systems of equations of the original CGN penalty approach appears in the work of Nordström, and Carpenter [26], and extension of the new general penalties presented herein is in principle analogous. Although it is straightforward to generalize many of the new penalty terms, those involving $\mathcal{D}^{T}$ require special consideration. As such, the generalization to systems if is a topic of current investigation. Extension to nonlinear equations has not been attempted, although it is likely that transforming weak form (primal or flux) FEM methods into strong form will provide valuable insight. 


\section{Conclusions}

A new approach is presented that generalizes the original multi-domain, summation-by-parts (SBP), penalty technique of Carpenter et al. [8] to include penalties on the solution and the first $p$ interface derivatives. The energy method is used to prove for $p \leq 2$ that the combined interior/interface operators are $L_{2}$ stable and conservative when used to discretize the 1D, linear Burgers equation. A discrete Sobolev inequality is then used to establish pointwise stability. The extension to 3D is conceptually "trivial" using tensor product algebra.

It is shown that the new interface procedure is applicable to two distinct sets of variables. The first implementation is reminiscent of the primal form method developed by Baumann and Oden, [4], while the second resembles the flux form LDG methods developed by Cockburn and Shu [11]. Although both formulations are $L_{2}$ and pointwise stable, and conservative, their proofs involve different variables. There are similarities, however. Indeed, the flux formulation can be implemented using the primal variables if the penalty parameters are allowed to vary with grid resolution. Furthermore, all stable flux formulations are also stable in the primal variables.

There are potential costs for elaborate penalties in either the primal or flux formulations. Penalizing data that is of inadequate accuracy can theoretically degrade the formal global accuracy of the method. Penalties built from second and higher derivatives are theoretically ill advised for this reason. A further potential cost is the degree to which some penalties increase the condition number of the resulting discretization matrix, making them unacceptably expensive for explicit temporal methods.

Extensive numerical experiments on the advection and the advection diffusion (linear Burgers') equations are used to verify the theoretical predictions. All simulations were performed using penalties limited to $p \leq 1$, i.e. the solution and first derivative. Two classes of of high-order SBP operators are used in these studies: 1) central finite difference, and 2) Legendre spectral collocation. General trends identified in these studies include the following: 1) The theoretical predictions are not always sharp. For example, finite difference simulations achieve design order convergence for all permutations of penalty parameters. Similarly, Legendre simulations using the primal formulation achieve optimal convergence for odd polynomial orders. Both these results are in contradiction to theoretical estimates. 2) The flux formulation is less susceptible to order reduction, particularly when using the Legendre spectral collocation methods. Thus, it can potentially benefit from a wide variety of penalty terms. Conversely, the suboptimal formal accuracy was observed when using the primal formulation for Legendre simulations.

\section{References}

[1] Abarbanel, S., Chertock, A. E., Strict stability of high-order compact difference schemes: The role of boundary conditions for hyperbolic pde's, I, (and II), J. Comput. Phy, Vol. 160, (2000), pp. 42-66.

[2] Arnold, D. N., Brezzi, F., Cockburn, B. and Marini, L. D., Discontinuous Galerkin methods for elliptic problems, Discontinuous Galerkin Methods. Theory, Computation and Applications, B. Cockburn, G. E. Karniadakis, and C.-W. Shu, eds., Lecture Notes in Comput. Sci. Engrg. Vol. 11, Springer-Verlag, New York, (2000), pp. 89-101.

[3] Arnold, D. N., Brezzi, F., Cockburn, B. and Marini, L. D., Unified analysis of discontinuous Galerkin methods for elliptic problems, SIAM J. Numer. Anal., Vol. 39, No. 5, (2002), pp. 1749-1779.

[4] Baumann, C. E., And Oden, J. T., A Discontinuous hp finite element method for the Euler and Navier-Stokes equations I. J. Numer. Methods Fluids, Vol. 175, (1999), pp. 331-341.

[5] Carpenter, M.H. Kennedy, C.A., Fourth-Order 2N-Storage Runge-Kutta schemes, NASA-TM-109111, April 1994.

[6] Carpenter, M.H, Gottlieb, D. and Abarbanel, S., Time-stable boundary conditions for finite difference schemes solving hyperbolic systems: methodology and application to high-order compact schemes, J. Comput. Phys., Vol. 111, No. 2, 1994.

[7] Carpenter, M.H., Gottlieb, D., Spectral methods on arbitrary grids, J. Comput. Phys., Vol. 129, (1996), pp. 74-86. 
[8] Carpenter, M.H., Nordström, J. and Gottlieb, D., A stable and conservative interface treatment of arbitrary spatial accuracy, J. Comput. Phys., Vol. 148, pp. 341-365, (1999).

[9] Carpenter, M.H., Nordström, J. and Gottlieb, D., Revisiting and extending interface penalties for multidomain summation-by-parts operators, NASA/TM-2007-214892, (2007).

[10] Chesshire, G., Henshaw, W. D. Composite overlapping meshes for the solution of partial differential equations, J. Comput. Phys., Vol. 90, (1990), pp. 1-64.

[11] Cockburn, B. And Shu, C.-W., The local discontinuous Galerkin method for time-dependent convectiondiffusion systems, SIAM J. Numer. Anal., Vol. 35, (1998), pp. 2440-2463.

[12] Don, W.S., Gottlieb, D., The Chebyshev-Legendre method: implementing Legendre methods on Chebyshev points, SIAM J. Num. Anal, Vol. 31, No. 6, (Dec., 1994), pp. 1519-1534.

[13] Diener, P., Dorband, E. N., Schnetter, E. And Tiglio, M. J. Sci. Comput., Vol. 32, No. 1, (July 2007), pp. 109-145.

[14] Gustafsson, B., The convergence rate for difference approximations to mixed initial boundary value problems, Math. Comp. Vol. 29 (1975), pp. 396-406.

[15] Hesthaven, J.S. and Gottlieb, D., A stable penalty method for the compressible Navier-Stokes equations: I. open boundary conditions, SIAM J. Sci. Comput., Vol. 17, 3, pp.579-612, 1996.

[16] Hesthaven, J.S., A stable penalty method for the compressible Navier-Stokes equations: II. One-dimensional domain decomposition schemes, SIAM J. Sci. Comput., Vol. 18, 3, 1997.

[17] Hesthaven, J. S., Teng, C. H.., Stable spectral methods on tetrahedral elements, SIAM J. Sci. Comput., Vol. 21, (2000), pp. 2352-2380.

[18] Hesthaven, J. S., Warburton, T., Nodal high-order methods on unstructured grids I. Time-domain solution of Maxwell's equations, J. Comput. Phys., Vol. 181, (2002), pp. 186-221.

[19] Hesthaven, J. S., Warburton, T., Nodal high-order methods on unstructured grids II. nonlinear problems, J. Comput. Phys.

[20] Kreiss, H.O. And Scherer, G., Finite element and finite difference methods for hyperbolic partial differential equations, Mathematical Aspects of Finite Elements in Partial Differential Equations, Academic Press, New York, 1974.

[21] Kreiss, H.O. and Lorenz, J., Initial-Boundary value problems and the Navier-Stokes Equation, Academic Press, Inc, 1989.

[22] P. D. Lax, B. Wendroff, Systems of Conservation Laws, Comm. Pure Appl. Math, 13, (1960), pp. 217-237.

[23] Mattsson, K. Boundary Procedures for Summation-by-Parts Operators, J. Sci. Comput., Vol. 18, No. 1, (Feb. 2003), pp 133-153.

[24] Mattsson, K., Svärd, M., Nordström, J, Stable and accurate artificial dissipation, J. Sci. Comput., Vol. 21, No. 1, (Aug. 2004), pp. 57-79.

[25] Mattsson, K., Nordström, J, Summation by parts operators for finite difference approximations of second derivatives, J. Comput. Phy., Vol. 199, No. 2, (Sept. 2004), pp. 503-540.

[26] Nordström, J., CArpenter, M. H., Boundary and interface conditions for high-order finite-difference methods Applied to the Euler and Navier-Stokes Equations J. Comput. Phy., Vol. 148, (1999), pp. 621-645.

[27] Nordström, J., Gustafsson, R., High Order Finite Difference Approximations of Electromagnetic Wave Propagation Close to Material Discontinuities, J. Sci. Comput., Vol. 18, No. 2, 2003.

[28] Olsson, P., Summation by parts, projections, and stability I, Math. Comp., Vol. 64, pp. 1035-1065, 1995.

[29] Olsson, P., Summation by parts, projections, and stability II, Math. Comp., Vol. 64, pp. 1473-1493, 1995. 
[30] Shu, C.-W., Different formulations of the discontinuous Galerkin method for the viscous terms, in Proceedings of Conference in Honor of Professor H.-C. Huang on the Occasion of His Retirement, W. M. Xui, Z. C. Shi, M. $\mathrm{Mu}$, and J. Zou, eds., Science Press, New York, Beijing, 2000, pp. 14-45.

[31] Strand, B., Summation by parts for finite difference approximations for d/dx, J. Comput. Phys., Vol. 110, No. 1, pp. 47-67, 1994.

[32] Strand, B., High-order difference approximations for hyperbolic initial boundary value problems, PhD Thesis, Uppsala University, Department of Scientific Computing, (1996).

[33] SvÄRD, M., On Coordinate transformations for Summation-by-parts Operators, J. Sci. Comput., Vol. 20, No. 1, (Feb. 2004), pp. 29-42.

[34] Svärd, M., AND Nordström, J., On the order of accuracy for difference approximations of initial-boundary value problems, J. Comput. Phys., Vol. 218, pp. 333-352, 2006.

[35] Third-order energy stable WENO scheme, J. Comput. Phys., Vol. 228, pp. 3025-3047, 2009. 


\begin{tabular}{|c|c|c|c|c|c|c|c|c|c|c|c|c|}
\hline \multirow{3}{*}{$\begin{array}{l}\text { Penalty } \\
\text { Parameters } \\
\text { and Norms } \\
\end{array}$} & \multicolumn{12}{|c|}{ Inflow-Outflow : Nonuniform } \\
\hline & \multicolumn{4}{|c|}{$\gamma=0.000$} & \multicolumn{4}{|c|}{$\gamma=0.010$} & \multicolumn{4}{|c|}{$\gamma=0.025$} \\
\hline & $\mathrm{p}=2$ & $\mathrm{p}=3$ & $\mathrm{p}=4$ & $\mathrm{p}=5$ & $\mathrm{p}=2$ & $\mathrm{p}=3$ & $\mathrm{p}=4$ & $\mathrm{p}=5$ & $\mathrm{p}=2$ & $\mathrm{p}=3$ & $\mathrm{p}=4$ & $\mathrm{p}=5$ \\
\hline \multicolumn{13}{|l|}{$\alpha=0 ; \beta=0$} \\
\hline$\|U-u\|_{2}$ & -2.00 & -3.01 & -4.00 & -5.02 & -2.06 & -2.96 & -3.95 & -6.87 & -2.08 & -3.02 & -4.99 & -7.34 \\
\hline$\|U-u\|_{\infty}$ & -2.01 & -2.98 & -4.00 & -5.09 & -1.88 & -3.09 & -4.28 & -7.33 & -1.91 & -3.20 & -4.64 & -7.54 \\
\hline$\|U-u\|_{\mathcal{P}}$ & -1.93 & -3.12 & -3.97 & -5.15 & -3.35 & -2.32 & -5.10 & -5.98 & -3.36 & -2.66 & -4.74 & -6.04 \\
\hline \multicolumn{13}{|l|}{$\alpha=0 ; \beta=\frac{1}{4}$} \\
\hline$\|U-u\|_{2}$ & -1.97 & -3.02 & -3.97 & -5.00 & -2.20 & -3.12 & -4.11 & -4.99 & -2.25 & -3.11 & -4.20 & -5.41 \\
\hline$\|U-u\|_{\infty}$ & -1.88 & -3.14 & -3.99 & -5.04 & -2.09 & -3.11 & -4.00 & -5.40 & -2.30 & -3.06 & -4.37 & -5.30 \\
\hline$\|U-u\|_{\mathcal{P}}$ & -1.84 & -3.21 & -3.93 & -5.32 & -1.98 & -3.23 & -4.05 & -5.18 & -2.31 & -3.21 & -4.15 & -5.20 \\
\hline \multicolumn{13}{|l|}{$\alpha=1 ; \beta=0$} \\
\hline$\|U-u\|_{2}$ & -3.01 & -4.00 & -4.93 & -6.03 & -2.57 & -3.58 & -4.68 & -5.55 & -2.57 & -3.60 & -4.51 & -5.49 \\
\hline$\|U-u\|_{\infty}$ & -3.01 & -3.95 & -4.92 & -6.01 & -2.35 & -3.91 & -4.70 & -5.45 & -2.35 & -4.06 & -4.63 & -5.22 \\
\hline$\|U-u\|_{\mathcal{P}}$ & -4.04 & -4.01 & -6.01 & -5.95 & -3.04 & -4.21 & -6.84 & -6.36 & -3.04 & -4.22 & -5.38 & -6.43 \\
\hline \multicolumn{13}{|l|}{$\alpha=1 ; \beta=\frac{1}{4}$} \\
\hline$\|U-u\|_{2}$ & -1.96 & -3.02 & -3.98 & -4.99 & -2.19 & -3.12 & -4.10 & -4.99 & -2.25 & -3.10 & -4.20 & -5.43 \\
\hline$\|U-u\|_{\infty}$ & -1.90 & -3.03 & -3.99 & -5.04 & -2.08 & -3.11 & -3.99 & -5.39 & -2.18 & -3.05 & -4.31 & -5.28 \\
\hline$\|U-u\|_{\mathcal{P}}$ & -1.84 & -3.20 & -3.93 & -5.32 & -1.97 & -3.23 & -4.04 & -5.18 & -2.20 & -3.21 & -4.11 & -5.22 \\
\hline
\end{tabular}

Table 1: Convergence Study of the Linear Wave Equation comparing the effects of $\gamma(\epsilon=0)$. Plotted are three norms of convergence rate, obtained using polynomials of order $p$ for $p=2, \cdots, p=5$, as functions of interface parameter combinations. 


\begin{tabular}{|c|c|c|c|c|c|c|c|c|c|c|c|c|}
\hline$\overline{\text { Penalty }}$ & \multicolumn{12}{|c|}{ Inflow-Outflow, Nonuniform Domain } \\
\hline Parameters & \multicolumn{4}{|c|}{$\epsilon=1.00$} & \multicolumn{4}{|c|}{$\epsilon=0.10$} & \multicolumn{4}{|c|}{$\epsilon=0.01$} \\
\hline and Norms & $\mathrm{p}=2$ & $\mathrm{p}=3$ & $\mathrm{p}=4$ & $\mathrm{p}=5$ & $\mathrm{p}=2$ & $\mathrm{p}=3$ & $\mathrm{p}=4$ & $\mathrm{p}=5$ & $\mathrm{p}=2$ & $\mathrm{p}=3$ & $\mathrm{p}=4$ & $\mathrm{p}=5$ \\
\hline \multicolumn{13}{|l|}{$\alpha=0 ; \beta=0$} \\
\hline$\|U-u\|_{2}$ & -1.98 & -4.01 & -4.00 & -5.84 & -1.96 & -3.98 & -4.04 & -6.01 & -2.39 & -3.74 & -4.95 & -5.92 \\
\hline$\|U-u\|_{\infty}$ & -1.97 & -4.01 & -4.01 & -5.85 & -2.01 & -3.98 & -4.09 & -6.01 & -2.90 & -3.69 & -5.20 & -5.88 \\
\hline$\left\|\mathcal{D} U-u_{x}\right\|_{2}$ & -1.99 & -3.00 & -4.00 & -4.99 & -1.99 & -3.00 & -3.99 & -4.99 & -1.94 & -3.10 & -4.14 & -5.15 \\
\hline$\overline{\|U-u\|_{\mathcal{P}}}$ & -1.99 & -4.03 & -4.01 & -5.91 & -1.94 & -3.99 & -3.97 & -6.00 & -1.70 & -3.70 & -4.13 & -5.80 \\
\hline \multicolumn{13}{|l|}{$\alpha=0 ; \beta=\frac{1}{4}$} \\
\hline$\|U-u\|_{2}$ & -2.01 & $\begin{array}{l}-3.02 \\
\end{array}$ & -4.02 & -5.12 & -2.98 & -3.36 & -4.85 & -5.56 & -3.38 & -4.38 & $\begin{array}{c}-5.20 \\
\end{array}$ & -6.40 \\
\hline$\|U-u\|_{\infty}$ & -2.01 & $\begin{array}{l}-3.02 \\
\end{array}$ & -4.02 & -5.08 & -2.75 & -4.09 & -4.99 & -5.96 & -2.95 & -4.47 & -4.81 & -6.54 \\
\hline$\left\|\mathcal{D} U-u_{x}\right\|_{2}$ & -2.02 & -3.00 & -4.01 & -4.99 & -2.04 & -3.02 & -4.00 & -5.00 & -2.44 & -3.33 & -4.22 & -5.26 \\
\hline$\|U-u\|_{\mathcal{P}}$ & -2.03 & -5.01 & -4.04 & -6.42 & -2.03 & -4.36 & -3.78 & -6.17 & -1.63 & -4.27 & -4.49 & -6.37 \\
\hline \multicolumn{13}{|l|}{$\alpha=1 ; \beta=0$} \\
\hline$\|U-u\|_{2}$ & -1.96 & -4.00 & -4.00 & -5.83 & -1.80 & -3.90 & -4.00 & -5.96 & -2.30 & -3.47 & -4.70 & -5.69 \\
\hline$\|U-u\|_{\infty}$ & -1.96 & -4.00 & -4.00 & -5.84 & -1.85 & -3.90 & -4.06 & -5.95 & -2.74 & -3.41 & -4.87 & -5.64 \\
\hline$\left\|\mathcal{D} U-u_{x}\right\|_{2}$ & -1.98 & -3.00 & -4.00 & -4.99 & -1.94 & -2.98 & -3.99 & -4.98 & -1.93 & -3.07 & -4.12 & -5.15 \\
\hline$\|U-u\|_{\mathcal{P}}$ & -1.97 & -4.02 & -4.01 & -5.91 & -1.78 & -3.92 & -3.94 & -5.95 & -1.69 & -3.61 & -3.88 & -5.62 \\
\hline \multicolumn{13}{|l|}{$\alpha=1 ; \beta=\frac{1}{4}$} \\
\hline$\|U-u\|_{2}$ & -2.00 & -3.00 & -4.01 & -5.09 & -2.97 & -3.36 & -4.85 & -5.55 & -3.37 & -4.38 & -5.20 & -6.40 \\
\hline$\|U-u\|_{\infty}$ & -2.00 & -3.01 & -4.00 & -5.04 & -2.73 & -4.08 & -4.99 & -5.96 & -2.95 & -4.47 & -4.81 & -6.54 \\
\hline$\left\|\mathcal{D} U-u_{x}\right\|_{2}$ & -2.01 & -3.00 & -4.00 & -4.99 & -2.04 & -3.02 & -4.00 & -5.00 & -2.44 & -3.33 & -4.22 & -5.26 \\
\hline$\|U-u\|_{\mathcal{P}}$ & -2.02 & -5.01 & -4.00 & -6.41 & -2.01 & -4.35 & -3.78 & -6.17 & -1.59 & -4.27 & -4.49 & -6.37 \\
\hline
\end{tabular}

Table 2: Convergence Study: Effects of $\epsilon$ in the linear Burgers' equation, using the primal (B-O) formulation. Plotted are four norms of convergence rate, obtained using polynomials of order $p$ for $p=2, \cdots, p=5$, as functions of interface parameter combinations. 


\begin{tabular}{|c|c|c|c|c|c|c|c|c|c|c|c|c|}
\hline$\overline{\text { Penalty }}$ & \multicolumn{12}{|c|}{ Inflow-Outflow, Nonuniform Domain } \\
\hline Parameters & \multicolumn{4}{|c|}{$\epsilon=1.00$} & \multicolumn{4}{|c|}{$\epsilon=0.10$} & \multicolumn{4}{|c|}{$\epsilon=0.01$} \\
\hline and Norms & $\mathrm{p}=2$ & $\mathrm{p}=3$ & $\mathrm{p}=4$ & $\mathrm{p}=5$ & $\mathrm{p}=2$ & $\mathrm{p}=3$ & $\mathrm{p}=4$ & $\mathrm{p}=5$ & $\mathrm{p}=2$ & $\mathrm{p}=3$ & $\mathrm{p}=4$ & $\mathrm{p}=5$ \\
\hline \multicolumn{13}{|l|}{$\alpha=0 ; \beta=0$} \\
\hline$\|U-u\|_{2}$ & -2.97 & -4.07 & -4.96 & -5.84 & -2.88 & -3.89 & -4.94 & -5.92 & -3.42 & -4.24 & -5.37 & -6.10 \\
\hline$\|U-u\|_{\infty}$ & -2.96 & -3.99 & -4.99 & -5.85 & -2.59 & -3.85 & -4.75 & -5.87 & -3.43 & -4.12 & -5.22 & -5.86 \\
\hline$\left\|\mathcal{D} U-u_{x}\right\|_{2}$ & -2.01 & -3.03 & -4.02 & -5.08 & -2.02 & -3.03 & -4.03 & -5.08 & -2.28 & -3.41 & -4.35 & -5.45 \\
\hline$\|U-u\|_{\mathcal{P}}$ & -2.81 & -3.81 & -5.86 & -7.28 & -1.89 & -3.94 & -3.72 & -5.91 & -2.44 & -3.91 & -3.19 & -6.00 \\
\hline \multicolumn{13}{|l|}{$\alpha=0 ; \beta=\frac{1}{4}$} \\
\hline$\|U-u\|_{2}$ & -2.90 & -3.96 & -4.91 & -5.82 & -3.01 & -4.04 & -4.98 & -6.00 & -3.72 & -4.39 & -5.56 & -6.30 \\
\hline$\|U-u\|_{\infty}$ & -2.91 & $\begin{array}{l}-3.92 \\
\end{array}$ & $\begin{array}{c}-5.02 \\
\end{array}$ & -5.90 & $\begin{array}{l}-3.43 \\
\end{array}$ & -4.04 & -4.99 & $\begin{array}{l}-6.02 \\
\end{array}$ & -3.41 & -4.42 & -5.54 & -6.28 \\
\hline$\left\|\mathcal{D} U-u_{x}\right\|_{2}$ & -2.00 & -3.00 & -3.98 & -5.00 & -1.99 & -2.99 & -3.98 & -5.02 & -2.59 & -3.39 & -4.44 & -5.45 \\
\hline$\|U-u\|_{\mathcal{P}}$ & -2.61 & -3.84 & -5.62 & -6.50 & -0.33 & -4.21 & -4.57 & -6.08 & -2.42 & -4.25 & -4.10 & -6.51 \\
\hline \multicolumn{13}{|l|}{$\alpha=1 ; \beta=0$} \\
\hline$\|U-u\|_{2}$ & -2.95 & -4.06 & -4.95 & -5.84 & -2.81 & -3.82 & -4.91 & -5.88 & -3.18 & $\begin{array}{l}-3.99 \\
\end{array}$ & -5.10 & -5.67 \\
\hline$\|U-u\|_{\infty}$ & -2.93 & -3.98 & -4.98 & -5.84 & -2.46 & -3.78 & -4.69 & -5.83 & -3.33 & -3.89 & -4.98 & -5.67 \\
\hline$\left\|\mathcal{D} U-u_{x}\right\|_{2}$ & -2.01 & -3.03 & -4.02 & -5.08 & -2.01 & -3.02 & -4.01 & -5.05 & -2.01 & -3.12 & -4.09 & -5.13 \\
\hline$\|U-u\|_{\mathcal{P}}$ & -2.79 & -3.79 & -5.86 & -7.30 & -1.98 & -3.88 & -3.72 & -5.87 & -2.78 & -3.70 & -3.51 & -5.78 \\
\hline \multicolumn{13}{|l|}{$\alpha=1 ; \beta=\frac{1}{4}$} \\
\hline$\|U-u\|_{2}$ & -2.90 & -3.94 & -4.90 & -5.80 & -3.00 & -4.04 & -4.98 & -6.00 & -3.72 & -4.39 & -5.56 & -6.30 \\
\hline$\|U-u\|_{\infty}$ & -2.92 & -3.90 & -5.02 & -5.88 & -3.41 & -4.03 & -4.98 & -6.02 & -3.41 & -4.42 & -5.54 & -6.28 \\
\hline$\left\|\mathcal{D} U-u_{x}\right\|_{2}$ & -1.99 & -2.99 & -3.98 & -5.00 & -1.99 & -2.99 & -3.98 & -5.02 & -2.59 & -3.39 & -4.44 & -5.45 \\
\hline$\|U-u\|_{\mathcal{P}}$ & -2.59 & -3.79 & -5.63 & -6.52 & 2.18 & -4.20 & -4.56 & -6.07 & -2.41 & -4.25 & -4.10 & -6.50 \\
\hline
\end{tabular}

Table 3: Convergence Study: Effects of $\epsilon$ in the linear Burgers' equation, using the flux (LDG) formulation. Plotted are four norms of convergence rate, obtained using polynomials of order $p$ for $p=2, \cdots, p=5$, as functions of interface parameter combinations. 


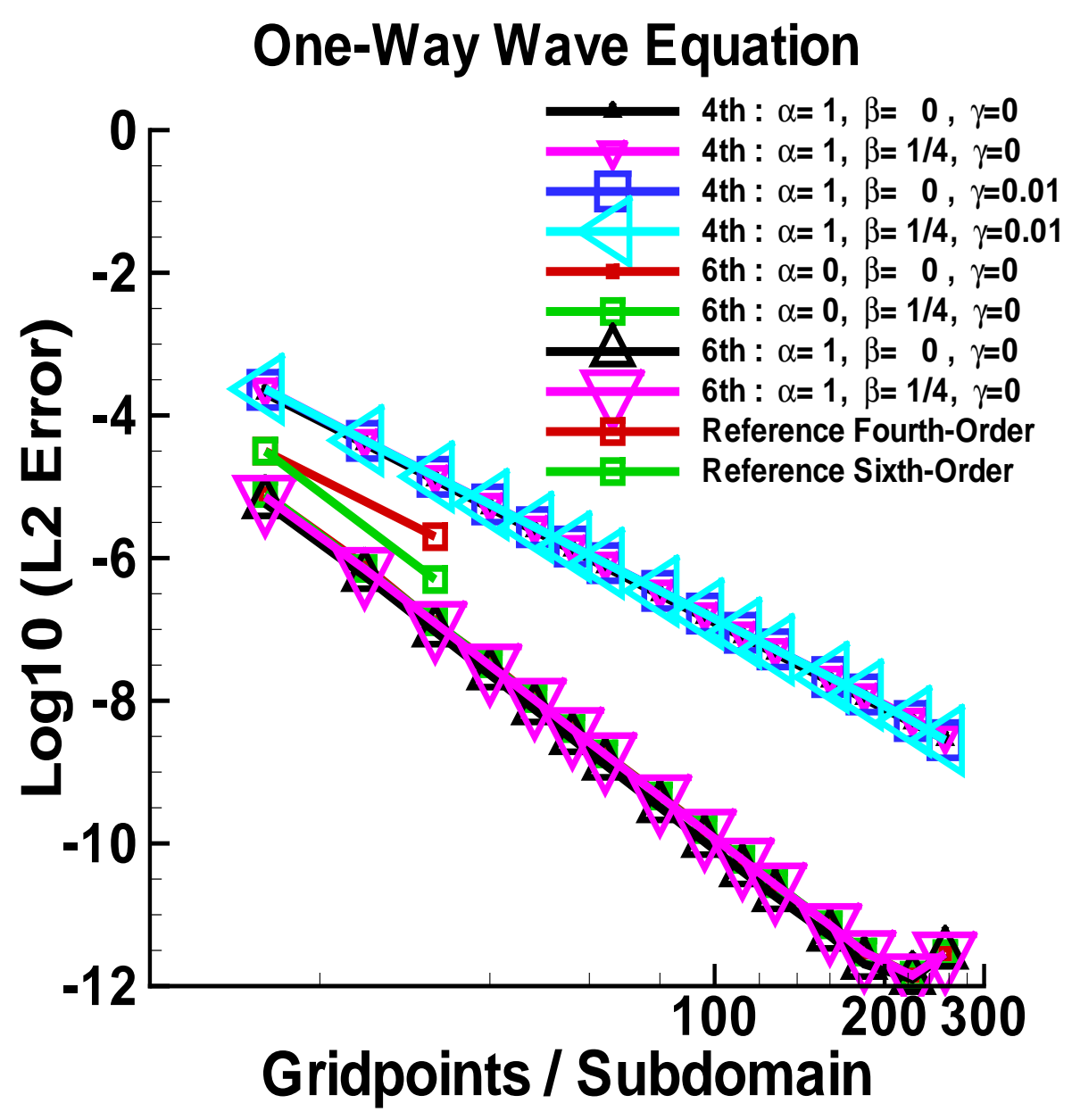

Figure 3: Comparison of $4^{\text {th }}$ - and $6^{\text {th }}$-order finite difference schemes on one-way wave equation. 


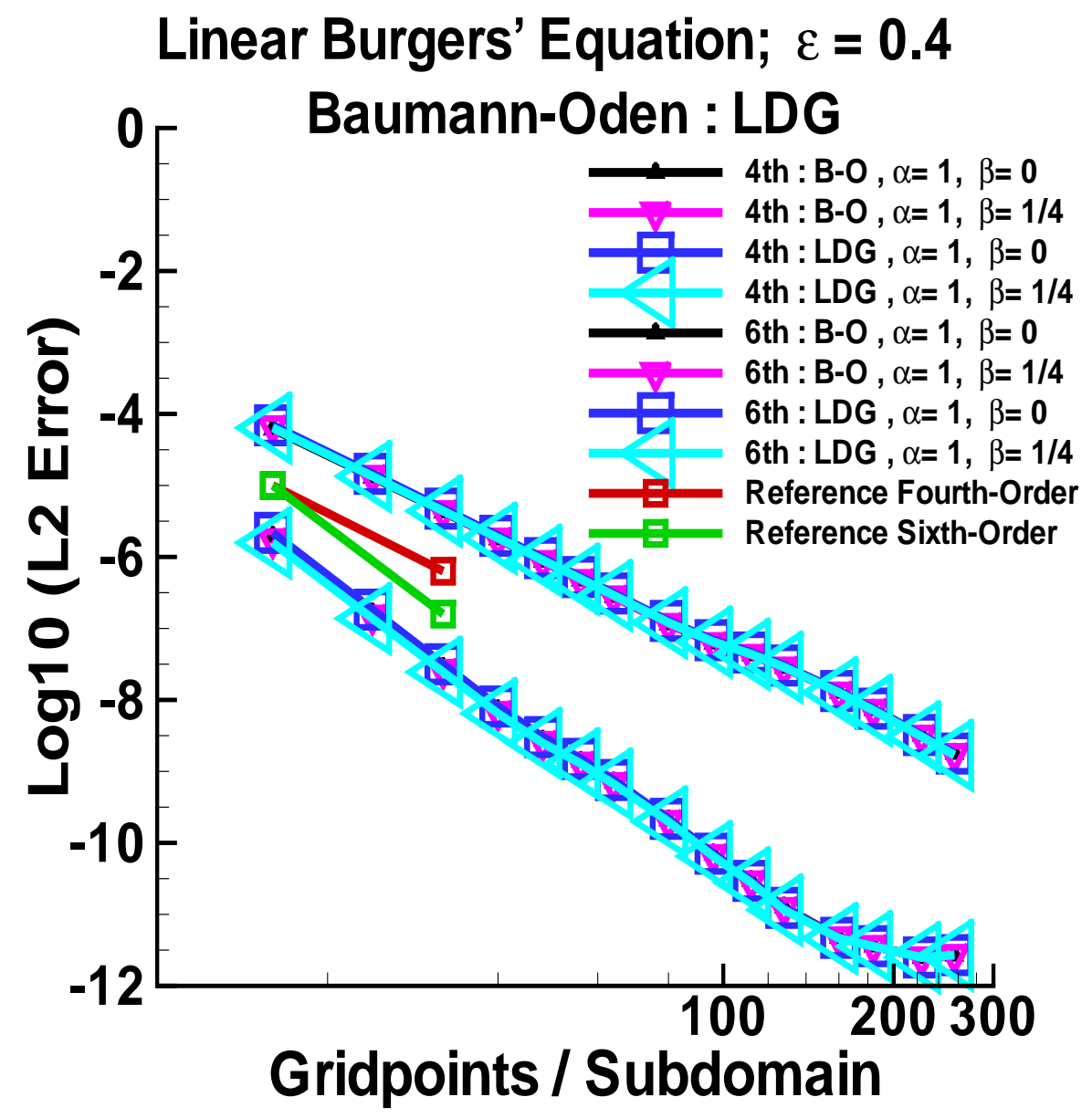

Figure 4: Comparison of $4^{\text {th }}$ - and $6^{\text {th }}$-order finite difference schemes on linear Burgers' equation. 


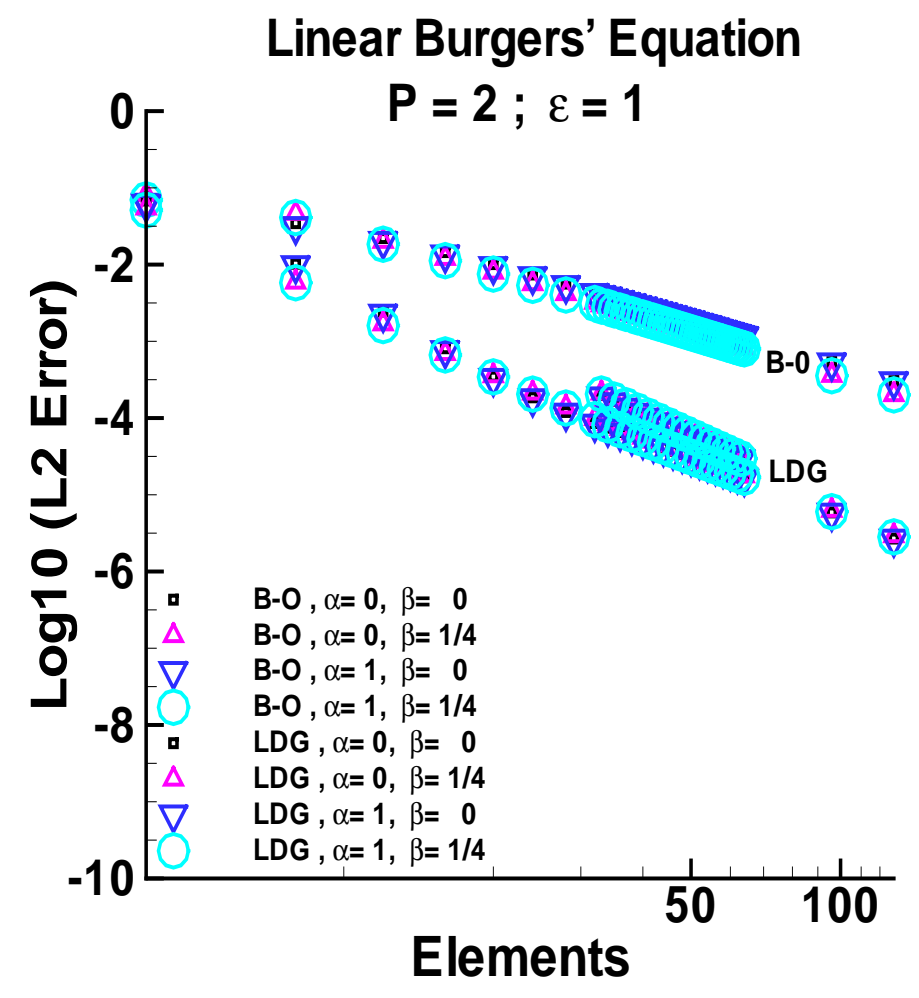

Figure 5: Comparison of Baumann-Oden and LDG schemes for multiple penalty parameters on the linear Burgers' equation: $P=2$.

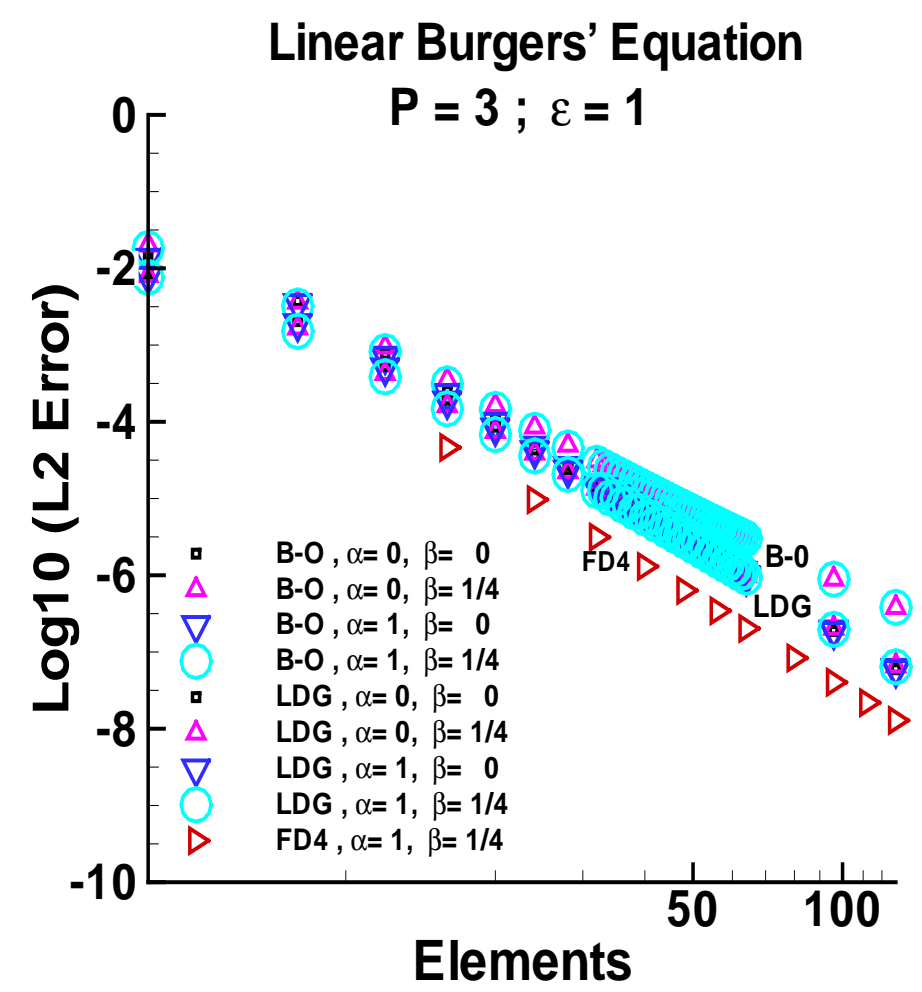

Figure 6: Comparison of Baumann-Oden and LDG schemes for multiple penalty parameters on the linear Burgers' equation: $P=3$. Also included is the $4^{t h}$-order finite difference result. 


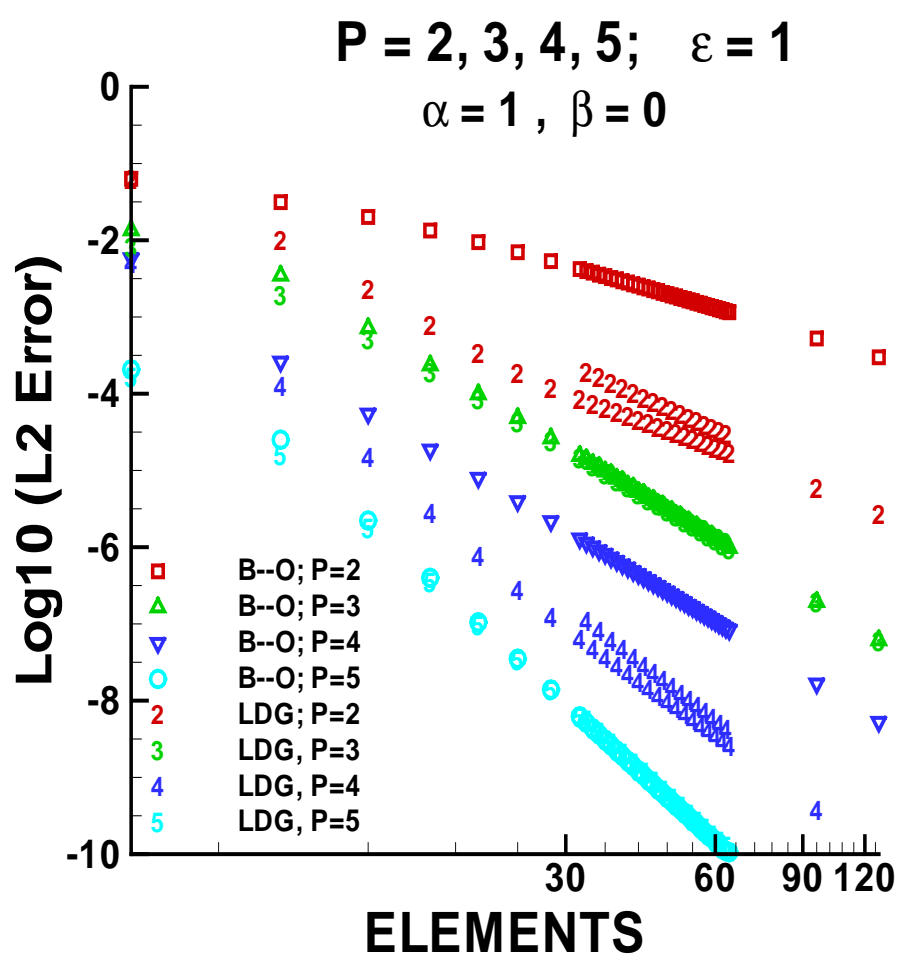

Figure 7: Comparison of Baumann-Oden and LDG schemes for multiple polynomial orders on the linear Burgers' equation: $\alpha=1, \beta=0$. 